\title{
Holes within galaxies: the egg or the hen?
}

\author{
R. Caimmi*
}

October 22, 2018

\begin{abstract}
Unsustained matter distributions unescapely collapse unless fragmentation and centrifugal or pressure support take place. Starting from the above evidence, supermassive compact objects at the centre of large-mass galaxies (defined as "holes") are conceived as the end-product of the gravitational collapse of local density maxima (defined as "central collapse") around which positive density perturbations (overdensities) are located. At the beginning of evolution, assumed to occur at recombination epoch, local density maxima are idealized as homogeneous peaks, while the surrounding envelopes are described by a power-law density profile, $\rho(r) \propto r^{b-3}, 0 \leq b \leq 3$, where $b=0$ represents a massless atmosphere and $b=3$ a homogeneous layer. The dependence of the density profile on a second parameter, chosen to be the ratio between peak and total (truncated) mass, $\kappa=M_{\mathrm{pk}} / M_{\mathrm{tr}}$, is analysed. Overdensity evolution is discussed in the context of quintessence cosmological models, which should be useful in dealing with the virialized phase. Aiming to describe the central collapse, further investigation is devoted to a special case where the quintessence effect is equivalent to additional curvature $(w=-1 / 3)$, and overdensities exhibit the selected density profile at recombination
\end{abstract}

*Astronomy Department, Padua Univ., Vicolo Osservatorio 2, I-35122 Padova, Italy email: roberto.caimmi@unipd.it 
epoch. A redshift-dependent, power-law relation between hole and (nonbaryonic) dark halo mass is used to express the dependence of the fractional mass, $\kappa$, on the overdensity mass, $M=M_{\mathrm{tr}}$, where the homogeneous peak and overdensity mass are related to the hole and dark halo mass, respectively. Computations are performed for a wide range of masses, $-1 \leq \log \left(M / \mathrm{M}_{10}\right) \leq 6$, and mean overdensity heights, $1 \leq \bar{\nu}_{i} \leq 4$, up to the end of central collapse, and density profiles of related configurations are determined together with additional parameters. The central collapse is completed in early times, no longer than a few hundredths of Gyr, which implies hole formation when proto-haloes, proto-bulges, and proto-disks are still relaxing. No appreciable change in evolution (up to the end of central collapse) is found with regard to different mean overdensity heights related to equal masses. On the other hand, it is recognized that homogeneous peaks collapse (in dimensionless coordinates) "faster" with respect to surroundings envelopes, in low-mass overdensities than in large-mass overdensities. In conclusion, it is inferred that gravitational collapse of homogeneous peaks within overdensities may be a viable mechanism for hole generation.

keywords - Dark matter; Dark energy; 95.35.+d; 95.36+x.

\section{Introduction}

The existence of large masses confined in a restricted central region of galaxies, was first suggested in order to explain short-period variability in brightness exhibited by quasars and active galactic nuclei (e.g., Terrel, 1964; Rees, 1966), and is widely supported by current high-resolution observations (for a review see e.g., Ferrarese and Ford, 2005; Merritt, 2006). There is an amount of increasing evidence, that compact objects at the centre of galaxies are supermassive black holes (e.g., Maor, 1998; Miller, 2006), but their origin is still debated.

As a first alternative, supermassive black holes are the end-product of compact (high-density) stellar systems which make galactic nuclei, where star encounters and star collisions are the dominant physical processes (Spitzer and Saslaw, 1966; Spitzer and Stone, 1967; Colgate, 1967; Sanders, 1970). Gravitational scattering (via star-star encounters) of energetic stars into elongated orbits, makes the density of the central region increase which, in turn, 
implies a higher rate of star evaporation, star collisions, star disruption, and star coalescence. The gas produced during (high-energy) star collisions, star disruption, and (high-mass) star death, falls to the central region and condenses into new stars which undergo further collisions. Accordingly, the end-product of the evolution of a dense nucleus appears to be the formation of a central, massive black hole, either by runaway star coalescence or gravitational collapse of a massive gas cloud (Begalman and Rees, 1978). The possibility of seeds for supermassive black holes which grow via accretion of stars and/or gas liberated by star collisions and star disruption, is also considered (Duncan and Shapiro, 1983; David et al., 1987a,b; Quinlan and Shapiro, 1987, 1989). Seeds for supermassive black holes could also be remnants of pop. III stars (e.g., Madau and Rees, 2001; Johnson and Bromm, 2007), or the (still speculative) intermediate-mass black holes which form in dense star cluster via merger events (e.g., Portegies Zwart et al., 2004; Maccarone et al., 2007). For further details, refer to Merritt (2006).

As a second alternative, supermassive black holes take origin before or during galaxy formation. In the spinar model (Morrison, 1969; Cavaliere et al., 1969; Cavaliere et al., 1970) the supermassive black hole progenitor is a single object (spinar). The spinar may be conceived as a "gravitational machine", where gravitational energy is converted into rotational energy via angular momentum conservation during the collapse phase, and rotational energy is converted into nonthermal electromagnetic energy (syncroton radiation) via acceleration of charged particles (mainly electrons) up to the relativistic regime, from magnetic torques due to the surface magnetic field. According to recent investigations, intermediate-mass black holes acting as seeds for supermassive black holes, could be formed from low-angular momentum material in primordial disks (Koushiappas et al., 2004) or isothermal collapse of atomic hydrogen gas $\left(M_{\mathrm{H}}=10^{7}-10^{9} \mathrm{~m}_{\odot}\right)$ within primordial dark matter haloes (Spaan and Silk, 2006).

The energy released by the formation and growth of supermassive black holes must have had a major impact on how gas cooled to form galaxies and galaxy clusters (Silk and Rees, 1998). In particular, accretion onto supermassive black holes provides the energy source for active galactic nuclei which, in turn, affects the evolution of galaxies (Silk, 2005). There is increasing evidence that supermassive black holes play an important role in the formation and global evolution of galaxies and of the intergalactic medium (Merloni et al., 2005). 
Seeds for pregalactic black holes could be found much earlier, related to topological defects during the inflation epoch, in particular closed domain walls (Rubin et al., 2001; Khlopov et al., 2002, 2005; for an extensive review, see Khlopov and Rubin, 2004). The resulting mass spectrum extends over a wide range, from superheavy to deeply subsolar black holes, where the upper limit follows from the condition that pieces of closed walls do not dominate within horizon before the whole wall enters it, while the lower limit is given by the condition that the black hole gravitational radius exceeds the width of contracting domain wall. In addition, low-mass objects are concentrated around their most massive counterparts within a cluster (e.g., Khlopov et al., 2005).

Seeds for supermassive black holes, or supermassive black holes, can form even in absence of baryons as an inevitable consequence of relativistic core collapse following the gravothermal catastrophe, under the restrictive assumption of self-interacting dark matter haloes (Balberg and Shapiro, 2002). The properties exhibited by self-interacting dark matter are in some ways intermediate between their counterparts related to hot and cold dark matter, respectively.

The current attempt starts with the working hypothesis, that supermassive black holes are the first structures formed within (sufficiently massive) density perturbations (hereafter quoted as "overdensities"). Similarly, about fifty years ago, galaxies were conceived as emerging from superdense primordial nuclei (Ambartsumian, 1958, 1965). In fact, unsustained matter distributions unescapely collapse unless fragmentation and centrifugal or pressure support take place. To this respect, the inner central region of collapsing proto-galaxies is a very special place, where both tidal effects and acquisition of angular momentum are minimized and may safely be thought of as negligible. Accordingly, both spherical symmetry and homogeneity are expected to be preserved, which implies the formation of a supermassive black hole, as in large-mass $\left(m>20-25 m_{\odot}\right)$ supernovae (e.g., Nomoto et al., 2005; Ohkube et al., 2006).

If (nonbaryonic) dark matter is mainly made of fermions (e.g., photinos), a fermion ball instead of a black hole could, in principle, take origin (e.g., Viollier, 1994; Munyaneza and Viollier, 2002; Munyaneza and Biermann, 2006) but no firm conclusion can be reached unless the nature of dark matter is known. Concerning ordinary matter, gravitational collapse can be halted by Fermi pressure for sufficiently low masses (white dwarfs, neutron stars, 
quark stars) but the contrary holds for higher masses, where the mean density of configurations close to the gravitational i.e. Schwartzschild radius is decreased. If a similar trend occurs for dark matter, and supermassive black holes are the result of dark matter collapse, a lower mass limit may safely be expected.

A short collapse time of inner, densest regions within overdensities, not exceeding a few hundredths of Gyr, would be consistent with the presence of a supermassive $\left(M=310^{9} \mathrm{~m}_{\odot}\right)$ black hole detected in the quasar SDSSJ $1148+5251$, at a redshift $z=6.41$ corresponding to a cosmic age $t=0.840$ Gyr (Willott et al., 2003).

Supermassive black hole formation via gravitational collapse of the local maxima around which overdensities are placed (hereafter quoted as "central collapse") is a viable scenario, to be tested when the nature and the properties of (nonbaryonic) dark matter are known. In this view, the current paper investigates the special configuration related to the end of central collapse, for different overdensity masses $\left(-1 \leq \log \left(M / \mathrm{M}_{10}\right) \leq 6 ; \mathrm{M}_{10}=10^{10} \mathrm{~m}_{\odot}\right)$ and mean peak heights $\left(1 \leq \bar{\nu}_{i} \leq 4\right)$, with assigned density profile at the beginning of evolution. Strictly speaking, a change of the quintessence equation of state parameter, $w$, and the quintessence degree of clustering, $\Gamma$, should be taken into consideration, in dealing with overdensity evolution. The general situation shall widely be discussed, to show how the above mentioned parameters could affect the virialized configuration. On the other hand, it will be shown that central collapse is completed in early times $(z>10)$ which makes the effect of quintessence negligible concerning both the equation of state parameter (e.g., Horellou and Berge, 2005) and the degree of clustering (e.g., Nunes and Mota, 2006). Accordingly, detailed calculations shall be limited to a special case where considerable simplification may be attained, overdensity virialization being outside the aim of the current attempt.

Some basic considerations are presented in Sect.2, where the assumed initial density profile is also defined. The initial overdensity configuration and related evolution up to the end of central collapse, are described in Sect. 3. The results are presented and discussed in Sects. 4 and 5, respectively. The conclusion makes the subject of Sect. 6 . 


\section{Basic considerations}

\subsection{The hole}

The existence of supermassive black holes at the centre of large-mass star spheroids, is supported with increasing evidence (e.g., Ferrarese and Ford, 2005; Merritt, 2006). For sake of brevity, supermassive compact objects within a restricted volume (in particular, supermassive black holes) shall be quoted as "holes". Accordingly, main components of a typical large-mass galaxy are: the (nonbaryonic) dark halo, the stellar halo, the disk, the bulge, and the hole. The hole, in turn, is surrounded by an accretion disk, which should be quoted as "vortex" or "mælmstrom", and a dust torus, with the emission of a jet along the polar directions during an active phase (e.g., Ferrarese and Ford, 2005).

\subsection{Black hole generation via gravitational collapse}

According to the density profile of their progenitor, black hole formation can occur bottom-up or top-down. To get more insight, let us refer to a spherical-symmetric, truncated power-law mass distribution, as:

$$
\begin{aligned}
& \frac{M(r)}{M_{\mathrm{tr}}}=\left(\frac{r}{R_{\mathrm{tr}}}\right)^{b} ; \\
& 0 \leq r \leq R_{\mathrm{tr}} ; \quad M_{\mathrm{tr}}=M\left(R_{\mathrm{tr}}\right) ; \quad 0 \leq b \leq 3 ;
\end{aligned}
$$

where $R_{\mathrm{tr}}$ is the truncation radius, $M_{\mathrm{tr}}$ the mass within the truncation radius, $b=0,1,3$ correspond to a Roche sphere (central mass point surrounded by a massless atmosphere), a (truncated) isothermal sphere, and a homogeneous sphere, respectively.

Matter distributions expressed by Eq. (1) are simple but nontrivial. For instance, models of baryonic collapse within dark matter haloes and formation of gaseous galactic disks involve use of truncated power-law mass distributions (Adams and Bloch, 2006).

The density profile related to Eq. (1) reads:

$$
\begin{array}{ll}
\frac{\rho(r)}{\rho_{\mathrm{tr}}}=\left(\frac{r}{R_{\mathrm{tr}}}\right)^{b-3} ; & 0 \leq r \leq R_{\mathrm{tr}} ; \\
\rho_{\mathrm{tr}}=\rho\left(R_{\mathrm{tr}}\right)=\frac{b}{3} \bar{\rho}_{\mathrm{tr}} ; & \bar{\rho}_{\mathrm{tr}}=\frac{3}{4 \pi} \frac{M_{\mathrm{tr}}}{R_{\mathrm{tr}}^{3}} ;
\end{array}
$$


where $\rho_{\text {tr }}$ and $\bar{\rho}_{\text {tr }}$ are the local density at the truncation radius and the mean density within the truncation radius, respectively. The central density cusp can be avoided by replacing an arbitrarily small inner core at $r=r_{\mathrm{pk}}$, with a homogeneous core where $\rho(r)=\rho\left(r_{\mathrm{pk}}\right), 0 \leq r \leq r_{\mathrm{pk}}$.

A spherical-symmetric matter distribution collapses into a black hole when the gravitational i.e. Schwartzschild radius (e.g., Landau and Lifchitz, 1966, Chap. XI, §97) is attained, as:

$$
R_{\mathrm{gr}}=\frac{2 G M_{\mathrm{tr}}}{c^{2}} ;
$$

where $G$ is the constant of gravitation and $c$ the light velocity in vacuum, to be intended as the light velocity in absence of baryonic matter. For further details, refer to Appendix A.

For the density profiles under discussion, the gravitational radius related to a sphere of radius, $r$, enclosing a mass, $M(r)$, is obtained by the combination of Eqs. (1) and (3), the latter generalized to a mass, $M(r)$. The result is:

$$
\frac{r_{\mathrm{gr}}}{R_{\mathrm{gr}}}=\left(\frac{r}{R}\right)^{b} ;
$$

where $R_{\mathrm{gr}}$ is the gravitational radius related to the truncation mass, according to Eq. (3).

In the special case of the isothermal sphere, $b=1$, all the spheres collapse into a black hole at the same time. Milder density profiles, $1<b \leq 3$, imply

$r_{\mathrm{gr}} / r<R_{\mathrm{gr}} / R_{\mathrm{tr}}$ i.e. top-down black hole formation: the gravitational radius is first attained at the truncation radius. Harder density profiles, $0 \leq b<1$, imply $r_{\mathrm{gr}} / r>R_{\mathrm{gr}} / R_{\mathrm{tr}}$ i.e. bottom-up black hole formation: the gravitational radius is first attained at the centre.

If holes are the end-products of overdensity central collapse, the formation mechanism must necessarily be bottom-up with regard to the whole mass, but all the possibilities remain open with regard to the local maximum, conceived as the proto-hole.

\subsection{Initial density profiles}

Power-law density profiles provide a poor fit to dark matter haloes unless sufficiently thin shells are considered. A better description relies on threeexponent laws with a central cusp (e.g., Hernquist, 1990; Navarro et al., 
1995, 1996; Zhao, 1996; Moore et al., 1998, 1999; Fukushige and Makino, 2001, 2003; Diemand et al., 2004; Reed et al., 2005; for the description of a fitting procedure see e.g., Caimmi and Marmo, 2003, 2004; Caimmi et al., 2005; Caimmi, 2006) or two-parameter laws (e.g., Sersic, 1968; Navarro et al., 2004; Merritt et al., 2005).

On the other hand, it is not the case at the beginning of the evolution which, for high-energy universes, may safely be related to recombination epoch (e.g., Caimmi, 1989), where the contribution of dark energy is still negligible. Aiming to describe overdensity evolution up to the end of the central collapse, a simple but nontrivial density profile is assumed.

More specifically, overdensities at recombination epoch $(z \approx 1100)$ are represented as homogeneous peaks surrounded by envelopes where the density profile is represented by a power-law, according to Eqs. (2). The explicit expression reads:

$$
\rho(r)=\left\{\begin{array}{ll}
\rho_{\mathrm{pk}} ; & 0 \leq r \leq r_{\mathrm{pk}} ; \\
\rho_{\mathrm{pk}}\left(\frac{r}{r_{\mathrm{pk}}}\right)^{b-3} ; & r_{\mathrm{pk}} \leq r \leq R_{\mathrm{tr}}
\end{array} ;\right.
$$

where $\rho_{\mathrm{pk}}$ and $r_{\mathrm{pk}}$ are the homogeneous peak density and radius, respectively.

Owing to spherical symmetry, the mass enclosed within the truncation surface is:

$$
M_{\mathrm{tr}}=\int_{0}^{R_{\mathrm{tr}}} \rho(r) 4 \pi r^{2} \mathrm{~d} r
$$

which may be integrated using Eq. (5). The result is:

$$
\begin{aligned}
& M_{\mathrm{tr}}=M_{\mathrm{pk}}\left\{1+\frac{3}{b}\left[\left(\frac{R_{\mathrm{tr}}}{r_{\mathrm{pk}}}\right)^{b}-1\right]\right\} ; \\
& M_{\mathrm{pk}}=\frac{4 \pi}{3} \rho_{\mathrm{pk}} r_{\mathrm{pk}}^{3} ;
\end{aligned}
$$

where $M_{\mathrm{pk}}$ is the homogeneous peak mass.

Using Eq. (7a), a dimensionless radius, $\Xi$, may be defined in terms of a dimensionless mass, $\kappa$, as:

$$
\begin{aligned}
& \Xi=\left(1+\frac{b}{3} \frac{1-\kappa}{\kappa}\right)^{1 / b} ; \\
& \Xi=\frac{R_{\mathrm{tr}}}{r_{\mathrm{pk}}} ; \quad \kappa=\frac{M_{\mathrm{pk}}}{M_{\mathrm{tr}}} ;
\end{aligned}
$$


and the mean density within the truncation radius, $\bar{\rho}_{\mathrm{tr}}=(3 / 4 \pi)\left(M_{\mathrm{tr}} / R_{\mathrm{tr}}^{3}\right)$, owing to Eqs. (7) and (8), takes the expression:

$$
\frac{\bar{\rho}_{\mathrm{tr}}}{\rho_{\mathrm{pk}}}=\Xi^{-3}\left[1+\frac{3}{b}\left(\Xi^{b}-1\right)\right] ;
$$

in terms of the dimensionless radius, $\Xi$, and:

$$
\frac{\bar{\rho}_{\mathrm{tr}}}{\rho_{\mathrm{pk}}}=\frac{1}{\kappa}\left[1+\frac{b}{3} \frac{1-\kappa}{\kappa}\right]^{-3 / b} ;
$$

in terms of the dimensionless mass, $\kappa$.

The combination of Eqs. (5) and (8) yields the local density at the truncation radius, as:

$$
\frac{\rho_{\mathrm{tr}}}{\rho_{\mathrm{pk}}}=\left[1+\frac{b}{3} \frac{1-\kappa}{\kappa}\right]^{(b-3) / b} ;
$$

and the combination of Eqs. (10) and (11) yields the local to global density ratio at the truncation radius, as:

$$
\frac{\rho_{\mathrm{tr}}}{\bar{\rho}_{\mathrm{tr}}}=\kappa\left[1+\frac{b}{3} \frac{1-\kappa}{\kappa}\right] ;
$$

which depend on two parameters, $b$ and $\kappa$.

With regard to a generic dimensionless radius, $\xi, \xi_{\mathrm{pk}} \leq \xi \leq \Xi$, Eqs. (7), (9), and (5) may be generalized as:

$$
\begin{aligned}
& M(r)=M_{\mathrm{pk}}\left[1+\frac{3}{b}\left(\xi^{b}-1\right)\right] ; \\
& \bar{\rho}(r)=\frac{\rho_{\mathrm{pk}}}{\xi^{3}}\left[1+\frac{3}{b}\left(\xi^{b}-1\right)\right] ; \\
& \rho(r)=\rho_{\mathrm{pk}} \xi^{b-3} ; \quad \xi=\frac{r}{r_{\mathrm{pk}}} ;
\end{aligned}
$$

and the combination of Eqs. (14) and (15) yields:

$$
\frac{\rho(r)}{\bar{\rho}(r)}=\left[\frac{3}{b}+\frac{b-3}{b} \xi^{-b}\right]^{-1} ;
$$

which is the generalization of Eq. (12) to a generic dimensionless radius, $\xi$, $\xi_{\mathrm{pk}} \leq \xi \leq \Xi$.

For a complete definition of density profiles within overdensities at recombination epoch, the boundary conditions derived from the cosmological model are needed. 


\section{Overdensity evolution up to the end of cen- tral collapse}

Let overdensities be made of a homogeneous peak surrounded by a heterogeneous envelope, at recombination epoch. The validity of the above mentioned assumption implies hole formation unless the collapse is halted by Fermi pressure, as in neutron star progenitors. In absence of any information about the (nonbaryonic) dark matter, hole formation via gravitational collapse cannot be escluded and must be taken into consideration. To this aim, overden-

sity evolution up to the end of central collapse shall be investigated in what follows.

Given a spherical-symmetric overdensity around a local maximum at the beginning of evolution, the central collapse is intended as the collapse of a dusty homogeneous sphere with same density and expansion rate with respect to the local maximum.

\subsection{Overdensities at recombination epoch}

The effect of dark energy may safely be neglected in early times $(z>10)$ and matter may be described, to a good extent after recombination, in the context of Friedmann universes with dust only. With regard to overdensities bound around a local density maximum, or peak (e.g., Heavens and Peacock, 1998), related mean and local values are defined as:

$$
\bar{\delta}=\frac{\bar{\rho}}{\rho_{h m}}-1 ; \quad \delta=\frac{\rho}{\rho_{h m}}-1 ;
$$

where $\rho_{h m}$ is the mean density of the matter Hubble flow. Keeping in mind the definition of mean density, the combination of Eqs. (17) yields (e.g., Ryden and Gunn, 1987):

$$
\bar{\delta}=\frac{1}{S} \iiint_{S} \delta \mathrm{d}^{3} S
$$

where $S$ is the overdensity volume.

For high-energy universes, as in current QCDM cosmologies where $\Omega_{m} \approx$ $0.3, \Omega_{q} \approx 0.7, \Omega_{m}+\Omega_{q}=1$, the initial configuration may safely be related to the recombination epoch (see e.g., Caimmi, 1989 for the special case of CDM cosmologies). According to Eq. (17), the overdensity mass reads:

$$
M=\left(1+\bar{\delta}_{i}\right) \rho_{h m}\left(a_{h i}\right) S_{i}=\left(1+\bar{\delta}_{i}\right) M_{h m} ;
$$


where $M_{h m}$ is the mass of matter Hubble flow enclosed within the volume, $S_{i}$, and the index, $i$, denotes recombination.

The mean overdensity value can be expressed in terms of the peak overdensity value, $\delta_{\mathrm{pk}}$, as:

$$
\bar{\delta}=F \delta_{\mathrm{pk}} ; \quad F=\frac{1}{S} \iiint_{S} \frac{\delta(\vec{r})}{\delta_{\mathrm{pk}}} \mathrm{d}^{3} S ;
$$

where the shape factor, $F$, lies in the range, $S_{\mathrm{pk}} / S \leq F \leq 1$, between the extreme situations of a peak surrounded by a massless atmosphere (lower limit) and by a layer of equal density (upper limit); and $S_{\mathrm{pk}}$ represents the volume filled by the peak, assumed to be homogeneous.

Let the mean and the local overdensity height be defined as the ratios:

$$
\bar{\nu}=\frac{\bar{\delta}}{\bar{\delta}_{\mathrm{M}}} ; \quad \nu=\frac{\delta}{\bar{\delta}_{\mathrm{M}}} ; \quad \bar{\delta}_{\mathrm{M}}=<\bar{\delta}^{2}>_{\mathrm{M}}^{1 / 2} ;
$$

where $\left\langle\bar{\delta}^{2}>_{\mathrm{M}}^{1 / 2}\right.$ is the rms overdensity value related to an assigned volume, $S$, at a given epoch (recombination in the case under discussion), and the index, $M$, means that a volume, $S$, within the Hubble flow, encloses a mass, $M=M_{h m}=\rho_{h m} S$. The combination of Eqs. (20) and (21) yields:

$$
\bar{\nu}=F \nu_{\mathrm{pk}} ; \quad \nu_{\mathrm{pk}}=\frac{\delta_{\mathrm{pk}}}{\bar{\delta}_{\mathrm{M}}} .
$$

If, in addition, the density profile is expressed by Eq. (5), the combination of Eqs. (14), (15), (16), and (17) yields:

$$
\begin{aligned}
& 1+\bar{\delta}(r)=\frac{1+\delta_{\mathrm{pk}}}{\xi^{3}}\left[1+\frac{3}{b}\left(\xi^{b}-1\right)\right] ; \\
& 1+\delta(r)=\left(1+\delta_{\mathrm{pk}}\right) \xi^{b-3} ; \\
& 1+\delta(r)=[1+\bar{\delta}(r)]\left[\frac{3}{b}+\frac{b-3}{b} \xi^{-b}\right]^{-1} ; \\
& \delta_{\mathrm{pk}}=\frac{\rho_{\mathrm{pk}}-\rho_{h m}}{\rho_{h m}} ; \quad 1 \leq \xi \leq \Xi ;
\end{aligned}
$$

and the particularization to the truncation radius, $\xi=\Xi$, by use of Eqs. (7a) and (8) produces:

$$
1+\bar{\delta}_{\mathrm{tr}}=\frac{1+\delta_{\mathrm{pk}}}{\kappa}\left[1+\frac{b}{3} \frac{1-\kappa}{\kappa}\right]^{-3 / b} ;
$$




$$
\begin{aligned}
& 1+\delta_{\mathrm{tr}}=\left(1+\delta_{\mathrm{pk}}\right)\left[1+\frac{b}{3} \frac{1-\kappa}{\kappa}\right]^{1-3 / b} ; \\
& 1+\delta_{\mathrm{tr}}=\left(1+\bar{\delta}_{\mathrm{tr}}\right) \kappa\left[1+\frac{b}{3} \frac{1-\kappa}{\kappa}\right] ; \\
& \lim _{b \rightarrow 0}\left[1+\frac{b}{3} \frac{1-\kappa}{\kappa}\right]^{1-3 / b}=\exp \left(-\frac{1-\kappa}{\kappa}\right) ; \\
& 0<\left[1+\frac{b}{3} \frac{1-\kappa}{\kappa}\right]^{1-3 / b} \leq 1 ; \quad 0<b \leq 3 ;
\end{aligned}
$$

accordingly, overdensities at recombination are defined by the following parameters: total mass, $M=M_{\text {tr }}$; mean value, $\bar{\delta}=\bar{\delta}_{\text {tr }}$; mean height, $\bar{\nu}=\bar{\nu}_{\text {tr }}$; fractional mass, $\kappa$; power-law density profile exponent, $b$; which allow the calculation of the peak overdensity, $\delta_{\mathrm{pk}}$, and the local overdensity, $\delta(r)$. At this stage, a cosmological model needs to be specified.

\subsection{Input parameters and cosmological model}

Overdensity masses and mean heights shall be considered in the range, $-1 \leq$ $\log \left(M / \mathrm{M}_{10}\right) \leq 6\left(\mathrm{M}_{10}=10^{10} \mathrm{~m}_{\odot}\right)$ and $1 \leq \bar{\nu} \leq 4$, respectively. Present-day rms overdensity values in the linear approximation, shall be assumed as in CDM cosmologies (e.g., Gunn, 1987; Ryden and Gunn, 1987). Related values at recombination may be determined, provided the temporal behaviour of the rms overdensity value for a selected mass, is known.

For QCDM cosmologies, it has still to be found an analytical solution which represents the growing mode of overdensities. In the special case of flat universes, $\Omega_{m}+\Omega_{q}=1$, and time-independent quintessence equation of state parameter, $w=$ const, the solution may be written in terms of the hypergeometric function, ${ }_{2} F_{1}$ (Silveria and Wega, 1994).

Extending the results related to the special case where the dark energy mimics a cosmological constant, $w=-1$ (Wang and Steinhardt, 1998; Carroll et al., 1992) to the range, $w>-1$ (Basilakos, 2003), and improving the results related to phantom energy, $w<-1$, yield the following approximation (Percival, 2005):

$$
\frac{\bar{\delta}_{\mathrm{M}}\left(a_{h}\right)}{\bar{\delta}_{\mathrm{M}}(1)}=\frac{5}{2} \frac{\Omega_{m}\left(a_{h}\right) a_{h}}{\left[\Omega_{m}\left(a_{h}\right)\right]^{u}-\Omega_{q}\left(a_{h}\right)+\left[1+0.5 \Omega_{m}\left(a_{h}\right)\right]\left[1+\lambda \Omega_{q}\left(a_{h}\right)\right]}
$$




$$
\begin{aligned}
& \Omega_{m}\left(a_{h}\right)=\frac{\Omega_{m} a_{h}^{-3}}{\Omega_{m} a_{h}^{-3}+\Omega_{q} a_{h}^{f\left(a_{h}\right)}} ; \\
& \Omega_{q}\left(a_{h}\right)=\frac{\Omega_{q} a_{h}^{f\left(a_{h}\right)}}{\Omega_{m} a_{h}^{-3}+\Omega_{q} a_{h}^{f\left(a_{h}\right)}} ; \\
& f\left(a_{h}\right)=-\frac{3}{\ln \left(a_{h}\right)} \int_{0}^{\ln \left(a_{h}\right)}[1+w(x)] \mathrm{d} \ln (x) ; \\
& \begin{array}{l}
u=\frac{3(1-w)}{5-6 w}\left\{1+\frac{1}{2} \frac{2-3 w}{(5-6 w)^{2}}\left[1-\Omega_{m}\left(a_{h}\right)\right]\right\} ; \\
\lambda=-\frac{1}{10} \frac{75 w+76}{25 w+2} ; \\
\Omega_{m}\left(a_{h}\right)+\Omega_{q}\left(a_{h}\right)=1 ; \quad \Omega_{m}=\Omega_{m}(1) ; \\
w\left(a_{h}\right)=\operatorname{const}=w ;
\end{array} \quad \Omega_{q}=\Omega_{q}(1) ;
\end{aligned}
$$

where $a_{h}$ is the cosmological scale factor, normalized to $a_{h}=1$ at the present time, $\Omega_{m}$ and $\Omega_{q}$ are density parameters related to matter (including radiation in the case under discussion, $z \lesssim 1100$ ) and quintessence, respectively. In the limit of constant quintessence equation of state parameter, Eq. (32d) reduces to (e.g., Horellou and Berge, 2005):

$$
f\left(a_{h}\right)=-3(1+w)
$$

according to Eq. (32h).

At recombination epoch, $z=1100$ to a good extent, and the cosmological scale factor reads:

$$
\left(a_{h}\right)_{i}=\frac{1}{1+z_{i}}=\frac{1}{1101}
$$

where the index, $i$, denotes the recombination.

The input parameters of the cosmological model are assumed to be the following (e.g., Manera and Mota, 2006):

$\Omega_{m}=0.3 ; \quad \Omega_{q}=0.7 ; \quad \Omega_{b}=0.047 ; \quad h=0.65 ; \quad \sigma_{8}=0.9 ;$

where $\Omega_{b}$ is the baryon density parameter, $h=H /\left(100 \mathrm{~km} \mathrm{~s}^{-1} \mathrm{Mpc}^{-1}\right)$ is the dimensionless Hubble parameter at $z=0$, and $\sigma_{8}=\bar{\delta}_{M(8)}$ is related to the mass, $M(8)$, within a sphere of radius, $R(8)=8 h^{-1} \mathrm{Mpc}$, filled by Hubble flow at present. 
A different dependence, $\sigma_{8}=f(w)$, is deduced according if X-ray cluster data (Wang and Steinhardt, 1998) or cosmic microwave background data (Doran et al., 2001) are used. The cases, $\sigma_{8}=0.8$ and $\sigma_{8}=0.7$ should also be considered (e.g., Horellou and Berge, 2005), but a complete investigation on this point is outside the aim of the current paper.

The system of measure, $\left[\mathrm{kpc} \mathrm{M}_{10} \mathrm{Gyr}\right], \mathrm{M}_{10}=10^{10} \mathrm{~m}_{\odot}$, shall be adopted in performing computations. The conversion formulae from and towards the standard astrophysical system of measure, $[\mathrm{cm} \mathrm{g} \mathrm{s}]$, are reported in Appendix B.

Using Eqs. (35), the current value of critical and mean matter density are:

$$
\begin{aligned}
& \rho_{\text {crit }}=\frac{3 H^{2}}{8 \pi G}=1.172610^{-8} \mathrm{M}_{10} \mathrm{kpc}^{-3} ; \\
& \rho_{\text {hm }}=\Omega_{m} \rho_{\text {crit }}=3.517810^{-9} \mathrm{M}_{10} \mathrm{kpc}^{-3} ;
\end{aligned}
$$

and the amount of matter Hubble flow enclosed within a sphere of radius, $R(8)=8 h^{-1} \mathrm{Mpc}$, is:

$$
M(8)=\frac{4 \pi}{3} \rho_{h m}\left(\frac{8}{h}\right)^{3}=94402 \mathrm{M}_{10} ;
$$

which is consistent with a value $M(8)=932513 \mathrm{M}_{10}$ deduced by use of a CDM cosmological model (Gunn, 1987). The rms overdensity spectrum at recombination epoch, $\bar{\delta}_{\mathrm{M}}\left(a_{h i}\right)$, shall be deduced from the above mentioned CDM model (e.g., Caimmi et al., 1990). Related present-day values in the linear theory, $\bar{\delta}_{\mathrm{M}}(1)$, are deduced from the standard relation (e.g., Zeldovich and Novikov, 1982):

$$
\rho_{h m}\left(a_{h}\right)=\rho_{h m}(1)(1+z)^{3} ;
$$

where $z$ is the redshift.

The radius of a sphere filled by matter Hubble flow of mass, $M=10^{\ell} \mathrm{M}_{10}$, is defined by the relation:

$$
M=10^{\ell} \mathrm{M}_{10}=\rho_{h m}\left(a_{h}\right) \frac{4 \pi}{3} R^{3}\left(a_{h}\right) ;
$$

and the combination of Eqs. (36) and (39) yields:

$$
R_{0}=R(1)=0.18932710^{(\ell+10) / 3} \mathrm{kpc} ;
$$


with regard to the current epoch, where $-1 \leq \ell \leq 6$ in the range of interest.

A linear dependence, $R \propto a_{h}$, implies the following:

$$
R\left(a_{h}\right)=\frac{R_{0}}{1+z}
$$

at any selected time; in particular, $R_{i}=R_{0} / 1101$ at recombination epoch.

For nonrotating, spherical-symmetric overdensities with null peculiar velocity field, the turnaround radius, $r_{\max }$, is defined by the relation (e.g., Peebles, 1980, Chap. II, §19A; Caimmi, 1989):

$$
\begin{aligned}
& \frac{R_{i}}{r_{\max }}=\Delta_{i} ; \\
& \Delta_{i}=1-\left(\Omega_{m}\right)_{i}^{-1}\left(1+\bar{\delta}_{i}\right)^{-1}>0 ;
\end{aligned}
$$

which holds for CDM universes. The combination of Eqs. (21) and (42) yields:

$$
\bar{\nu}_{i} r_{\max }=R_{i}\left(\bar{\delta}_{\mathrm{M} i}\right)^{-1} ; \quad\left|1-\left(\Omega_{m}\right)_{i}^{-1}\right| \ll \bar{\delta}_{i} \ll 1 ;
$$

to the first order in $\bar{\delta}_{i}$.

The rms overdensity value in the linear theory, $\bar{\delta}_{\mathrm{M} i}$, and the radius of the related sphere, $R$, are listed in Tab. 1 with regard to current and recombination epoch, using a CDM model (Gunn, 1987), as a function of the mass. The product, $\bar{\nu}_{i} r_{\max }$, deduced from Eq. (43), is also listed therein. Overdensity values at recombination epoch, $\bar{\delta}_{\mathrm{M} i}$, deduced from their present-day counterparts, $\bar{\delta}_{\mathrm{M} 0}$, by use of Eqs. (32), related to QCDM models with constant quintessence equation of state parameter, $w$, yield $z_{i}=1101.5263$ instead of the assumed value, $z_{i}=1100$, making a close agreement, as expected.

Turning to the general case of QCDM cosmologies, the dynamical expansion of the universe is described by the Friedmann equations (e.g., Percival, 2005):

$$
\begin{aligned}
& \frac{H^{2}\left(a_{h}\right)}{H_{0}^{2}}=\Omega_{m} a_{h}^{-3}+\Omega_{k} a_{h}^{-2}+\Omega_{q} a_{h}^{f\left(a_{h}\right)} ; \\
& \Omega_{k}=1-\Omega_{m}-\Omega_{q} ; \quad \Omega_{u}\left(a_{h}\right)=\frac{\rho_{u}\left(a_{h}\right)}{\rho_{\text {crit }}\left(a_{h}\right)} ; \quad u=m, q ; \\
& \rho_{\text {crit }}\left(a_{h}\right)=\frac{3 H^{2}\left(a_{h}\right)}{8 \pi G} ; \quad H\left(a_{h}\right)=\frac{\dot{a}_{h}}{a_{h}} ;
\end{aligned}
$$




\begin{tabular}{cccccc}
\hline \hline $\log \left(M / \mathrm{M}_{10}\right)$ & $\bar{\delta}_{\mathrm{M} 0}$ & $R_{0} / \mathrm{kpc}$ & $\bar{\delta}_{\mathrm{M} i}$ & $R_{i} / \mathrm{kpc}$ & $\bar{\nu}_{i} r_{\max }$ \\
\hline-1 & 14 & $1.89 \mathrm{E}+2$ & $1.27 \mathrm{E}-2$ & $1.72 \mathrm{E}-1$ & $8.13 \mathrm{E}+0$ \\
0 & 11 & $4.08 \mathrm{E}+2$ & $1.00 \mathrm{E}-2$ & $3.70 \mathrm{E}-1$ & $2.23 \mathrm{E}+1$ \\
1 & 8.0 & $8.79 \mathrm{E}+2$ & $7.26 \mathrm{E}-3$ & $7.98 \mathrm{E}-1$ & $6.60 \mathrm{E}+1$ \\
2 & 5.3 & $1.89 \mathrm{E}+3$ & $4.81 \mathrm{E}-3$ & $1.72 \mathrm{E}+0$ & $2.15 \mathrm{E}+2$ \\
3 & 3.4 & $4.08 \mathrm{E}+3$ & $3.08 \mathrm{E}-3$ & $3.70 \mathrm{E}+0$ & $7.21 \mathrm{E}+2$ \\
4 & 1.9 & $8.79 \mathrm{E}+3$ & $1.72 \mathrm{E}-3$ & $7.98 \mathrm{E}+0$ & $2.78 \mathrm{E}+3$ \\
5 & 0.87 & $1.89 \mathrm{E}+4$ & $7.89 \mathrm{E}-4$ & $1.72 \mathrm{E}+1$ & $1.31 \mathrm{E}+4$ \\
6 & 0.32 & $4.08 \mathrm{E}+4$ & $2.90 \mathrm{E}-4$ & $3.70 \mathrm{E}+1$ & $7.66 \mathrm{E}+4$ \\
\hline \hline
\end{tabular}

Table 1: The rms overdensity value in the linear theory, $\bar{\delta}_{\mathrm{M}}$, and the radius of the related sphere, $R$, with regard to current and recombination epoch, using a CDM model (Gunn, 1987), as a function of the mass. The product, $\bar{\nu}_{i} r_{\max }$, deduced from Eq. (43), is also listed. The current and recombination epoch are denoted by the indices, 0 and $i$, respectively. Overdensity values at recombination epoch, $\bar{\delta}_{\mathrm{M} i}$, deduced from their present-day counterparts, $\bar{\delta}_{\mathrm{M} 0}$, by use of Eqs. (32), related to QCDM models with constant quintessence equation of state parameter, $w$, yield $z_{i}=1101.5263$ instead of the assumed value, $z_{i}=1100$, making a close agreement, as expected. 
where $\Omega_{k}$ is the curvature density parameter, which is null for flat universes, and Eqs. (32) hold. The function, $f\left(a_{h}\right)$, is calculated by solving the conservation of energy equation for the dark energy (Caldwell et al., 1998a):

$$
\frac{\mathrm{d}\left(c^{2} \rho_{q} a_{h}^{3}\right)}{\mathrm{d} a_{h}}=-3 p_{q} a_{h}^{2}
$$

which yields:

$$
\rho_{q}\left(a_{h}\right)=\rho_{q} a_{h}^{f\left(a_{h}\right)}
$$

on the other hand, the conservation of matter mass produces:

$$
\rho_{m}\left(a_{h}\right)=\rho_{m} a_{h}^{-3}
$$

in the case under consideration of flat universes, the combination of Eqs. (44), (46), and (47) yields:

$$
\left(\frac{\dot{a}_{h}}{a_{h}}\right)^{2}=H^{2}\left(a_{h}\right)=\frac{8 \pi G}{3}\left[\rho_{m} a_{h}^{-3}+\rho_{q} a_{h}^{f\left(a_{h}\right)}\right] ;
$$

where the function, $f\left(a_{h}\right)$, is defined by Eq. (32d), or Eq. (33) in the limit of constant quintessence equation of state parameter, $w$.

Though the action of the dark energy may be explained in different ways (e.g., Caldwell et al., 1998a), the current interpretation is based on a dynamical evolving scalar field slowly rolling down its potential, $\mathcal{V}(\phi)$. If quintessence is minimally coupled to gravity i.e. any couplings to other fields are supposed to be negligibly small, the equation of motion for the scalar field within the Hubble flow is (e.g., Manera and Mota, 2006):

$$
\ddot{\phi}=-3 H \dot{\phi}-\frac{\mathrm{d} \mathcal{V}}{\mathrm{d} \phi}
$$

where the variable, $\phi$, may be conceived as a curvilinear coordinate (with the dimension of a length) which describes the evolution of the scalar field. A dynamical analogon of Eq. (49) is e.g., a solid shpere in free fall within a homogeneous fluid under the action of gravitation, where the first and the second term on the right-hand side of Eq. (49) are related to viscous and gravitational force, respectively. 
The pressure and the density of the scalar field are (e.g., Nunes and Mota, 2006):

$$
\begin{aligned}
& p_{q}=\frac{1}{2} \rho_{\mathrm{K}} \dot{\phi}^{2}-\rho_{\mathrm{P}} \mathcal{V}(\phi) ; \\
& \rho_{q}=\frac{1}{2} \frac{\rho_{\mathrm{K}}}{c^{2}} \dot{\phi}^{2}+\frac{\rho_{\mathrm{P}}}{c^{2}} \mathcal{V}(\phi) ;
\end{aligned}
$$

where $\rho_{\mathrm{K}}$ and $\rho_{\mathrm{P}}$ are mass densities related to kinetic and potential energy, respectively, which implies $\rho_{\mathrm{K}} \dot{\phi}^{2} / 2$ and $\rho_{\mathrm{P}} \mathcal{V}(\phi)$ are kinetic and potential energy densities, respectively, of the scalar field ${ }^{1}$. It is apparent that a slow rolling of the scalar field, $\rho_{\mathrm{K}} \dot{\phi}^{2} / 2 \ll \rho_{\mathrm{P}} \mathcal{V}(\phi)$, implies a negative pressure via Eq. (50).

The quintessence equation of state parameter, via Eqs. (50) and (51), reads:

$$
w=\frac{p_{q}}{c^{2} \rho_{q}}=\frac{\rho_{\mathrm{K}} \dot{\phi}^{2} / 2-\rho_{\mathrm{P}} \mathcal{V}(\phi)}{\rho_{\mathrm{K}} \dot{\phi}^{2} / 2+\rho_{\mathrm{P}} \mathcal{V}(\phi)} ;
$$

where, in general, $w$ changes in time. In the special case of a static scalar field, $\dot{\phi}=0, w=-1$, and the quintessence mimics the effect of a cosmological constant. In the special case of a steady rolling, $\dot{\phi}=$ const, and timeindependent potential, $\mathcal{V}(\phi)=(1 / s)\left(\rho_{\mathrm{K}} / \rho_{\mathrm{P}}\right)(\dot{\phi} / 2), s$ real number, Eq. $(52)$ reduces to: $w=(s-1) /(s+1)$, where $s=0,1 / 6,1 / 5,1 / 4,1 / 3,1 / 2,1$, yields $w=-1,-5 / 7,-2 / 3,-3 / 5,-1 / 2,-1 / 3,0$, respectively, $w$ is also time-independent, and the quintessence mimics the effect of an ecsessence (e.g., Iliev and Shapiro, 2001; Horellou and Berge, 2005), which is a perfect fluid (e.g., Nunes and Mota, 2006).

The general case, $w=w(t)$, can result from a changing ratio of quintessence kinetic to potential energy which, in turn, is owing to the evolution of the related scalar field potential (e.g., Caldwell et al., 1998a). Though timevarying equations of state are closer to the real situation (e.g., Wetterich, 1995; Amendola, 2000; Battye and Weller, 2003; Mota and van de Bruck, 2004; Percival, 2005; Manera and Mota, 2006; Nunes and Mota, 2006; Maio

\footnotetext{
${ }^{1}$ The mass densities, $\rho_{\mathrm{K}}$ and $\rho_{\mathrm{P}}$, are usually omitted in literature under the pretext that the chosen units imply unitary values of the light velocity in vacuum, $c=1 u_{c}$, and the Planck constant, $h=1 u_{h}$, where $u_{c}$ and $u_{h}$ are the unit velocity and the unit action, respectively, related to the chosen system of measure. In author's opinion, this practice is extremely dangerous, as the dimensions of the terms appearing in any equation cannot be tested.
} 
et al., 2006; Basilakos and Voglis, 2007), the special case of constant $w$ makes considerable simplification (e.g., Caldwell et al., 1998a; Wang and Steinhardt, 1998; Weinberg and Kamionkowski, 2003; Horellou and Berge, 2005; Maor and Lahav, 2005). Qualitatively, many of the effects of general $w(t)$ models can be predicted by interpolating between models with constant $w$. If the quintessence equation of state varies only slowly with time, the observational predictions are well approximated by treating $w(t)=$ const $=w$ (Wang et al., 2000; Percival, 2005). More realistic models where $w=w(t)$ such as 2EXP (e.g., Nunes and Mota, 2006) and SUGRA (e.g., Maio et al., 2006) show that $w=$ const to a good extent for $z \gtrsim 10$ or $t \lesssim 0.5$ Gyr.

In dealing with overdensity central collapse, time scales no longer than a few hudredths of Gyr are involved, or redshift substantially larger than about $z=10$. Accordingly, $w=$ const may safely be assumed in the current paper.

The combination of Eqs. (33), (46), (47) and (48) yields at recombination:

$$
\begin{aligned}
& \left(\frac{\dot{a}_{h i}}{a_{h i}}\right)^{2}=H^{2}\left(a_{h i}\right)=\frac{2 G}{R_{h i}^{3}}\left[M_{m h}+M_{q h}\left(a_{h i}\right)\right] ; \\
& M_{q h}\left(a_{h}\right)=\frac{4 \pi}{3} \rho_{q}\left(a_{h}\right) a_{h}^{3}=\frac{4 \pi}{3} \rho_{q} a_{h}^{-3 w} ;
\end{aligned}
$$

where $R_{h}$ is the radius of a sphere filled by Hubble flow of mass, $M_{m h}+$ $M_{q h}\left(a_{h}\right)$, and $M_{q}$ is formally defined as a quintessence "mass" (Caimmi, 2007). Let a few limiting but relevant situations be illustrated with more detail.

In the special case, $w=0, f\left(a_{h}\right)=-3$, according to Eq. (33), and the effect of quintessence is equivalent to the presence of additional matter, as shown by Eq. (44a). Nothing changes with respect to a CDM universe where $\left(\Omega_{m}\right)_{\mathrm{CDM}}=\Omega_{m}+\Omega_{q}$ (e.g., Caldwell et al., 1998a). The quintessence mass is time-independent via Eq. (53b).

In the special case, $w=-1 / 3, f\left(a_{h}\right)=-2$, according to Eq. (33), and the effect of quintessence is equivalent to the presence of additional curvature, as shown by Eq. (44a). Nothing changes with respect to a CDM universe where $\left(\Omega_{k}\right)_{\mathrm{CDM}}=\Omega_{k}+\Omega_{q}$ (e.g., Horellou and Berge, 2005). The quintessence mass scales as $a_{h}$ via Eq. (53b).

In the special case, $w=-1, f\left(a_{h}\right)=0$, according to Eq. (33), and the effect of quintessence is equivalent to the presence of a cosmological constant, 
as shown by Eq. (44a). Nothing changes with respect to a CDM universe in presence of a cosmological constant where $\Lambda /\left(3 H^{2}\right)=\Omega_{q}$ (e.g., Horellou and Berge, 2005). The quintessence mass scales as $a_{h}^{3}$ via Eq. (53b).

\subsection{Overdensity expansion and central collapse: gen- eral ideas}

The dynamics of nonlinear structure formation in the universe may show a distinct signature associated to the nature of the dark energy and a particular model (Mota and van de Bruck, 2004). In this view, the behaviour of quintessence during the nonlinear regime of structure formation, is conceived as lying between two limiting cases, namely (i) full clustering i.e. the scalar field responds to the infall in the same way as the matter, and (ii) unclustering i.e. the scalar field remains homogeneous and the sole effect is a tidal potential acting on the matter overdensity.

The general case of partial clustering can be taken into consideration, at the expense of a much more complicated continuity equation for the quintessence overdensity (e.g., Mota and van de Bruck, 2004; Maor and Lahav, 2005; Nunes and Mota, 2006). Even though it has been shown that quintessence cannot be perfectly smooth (Caldwell et al., 1998a,b), clustering is usually assumed to be negligible on scales less than about $100 \mathrm{Mpc}$ (e.g., Wang and Steinhardt, 1998; Weinberg and Kamionkowski, 2003; Battye and Weller, 2003; Horellou and Berge, 2005). It is therefore common practice to keep the quintessence homogeneous during the evolution of overdensities. The effects of relaxing this assumption were explored in recent attempts (e.g., Mota and van de Bruck, 2004; Percival, 2005; Manera and Mota, 2006; Nunes and Mota, 2006).

Given a spherical-symmetric overdensity and an infinitely thin spheri-

cal shell at a distance, $r$, from the centre, in the limit of fully clustered quintessence, the equation of motion is still expressed by Eq. (48) provided the Hubble parameter, $H$, is replaced by $H_{r}=\dot{r} / r$ (e.g., Nunes and Mota, 2006). In general, the evolution of the density of the scalar field (continuity equation) within the shell is (e.g., Mota and van de Bruck, 2004; Maor and Lahav, 2005; Nunes and Mota, 2006):

$$
\dot{\rho}_{q}+3 \frac{\dot{r}}{r}(1+w) \rho_{q}=\Gamma
$$


where $\Gamma$ describes the quintessence density change due to the cosmic expansion. In the limit of fully clustered quintessence, $\Gamma=0$ as for matter. In the limit of unclustered quintessence, $\Gamma=-3\left(\dot{a}_{h} / a_{h}-\dot{r} / r\right)(1+w) \rho_{q}$, and Eq. (54) reduces to its counterpart related to the quintessence Hubble flow.

The shell expansion is related to cosmic expansion and overdensity change, as (e.g., Nunes and Mota, 2006):

$$
\frac{\dot{r}}{r}=\frac{\dot{a}_{h}}{a_{h}}-\frac{1}{3} \frac{\dot{\delta}(r)}{1+\delta(r)}
$$

where $\delta(r)$ is the shell overdensity, defined by Eq. (17).

The real situation may safely be expected to lie between the above mentioned limiting cases, where the quintessence is clustering together with the matter and remains homogeneous, respectively (e.g., Maor and Lahav, 2005; Caimmi, 2007). In general, overdensities reach turnaround and collapse earlier in QCDM models with larger quintessence equation of state parameter, $w$, and vice versa (e.g., Weinberg and Kamionkowski, 2003; Horellou and Berge, 2005). On the other hand, the difference is small in early times, where the effect of the dark energy is still negligible (e.g., Weinberg and Kamionkowski, 2003; Horellou and Berge, 2005; Nunes and Mota, 2006).

For this reason, the quintessence equation of state parameter, $w$, shall be kept constant during overdensity evolution up to the end of central collapse, which is expected to occur in early times. The above condition, in turn, implies that different evolutions related to different degrees of quintessence are essentially indistinguishable except for the later stages when quintessence starts to dominate at low redshifts. In general, the evolution depends on the quintessence equation of state, the potential of the scalar field, and the quintessence contribution to the total energy budget of the universe at high redshifts. For further details, refer to e.g., Nunes and Mota (2006). Accordingly, the quintessence within overdensities shall be assumed as fully clustered to simplify the calculations.

Under the above mentioned restrictions, the density ratio of clustered to unclustered quintessence may be expressed in terms of the related overdensity value, as (e.g., Nunes and Mota, 2006):

$$
\frac{\rho_{q}(r)}{\rho_{q h}\left(a_{h}\right)}=\left[\frac{1+\delta(r)}{1+\delta\left(r_{i}\right)}\right]^{1+w} \quad ; \quad w=\text { const } ; \quad \Gamma=0 ;
$$


where $\rho_{q h}$ is the density within the quintessence Hubble flow and the index, $i$, denotes the beginning of evolution.

The integration of Eq. (54) where $w=$ const, $\Gamma=0$, yields (e.g., Caimmi, 2007):

$$
\frac{\rho_{q}(r)}{\rho_{q}\left(r_{\max }\right)}=\left(\frac{r}{r_{\max }}\right)^{-3(1+w)} ; \quad w=\mathrm{const} ; \quad \Gamma=0 ;
$$

with regard to the turnaround configuration, $r=r_{\max }$. Accordingly, the quintessence density is related to the radius by a power law with exponent, $b-3=-3 w-3$. The special case, $w=-1 / 3, b=1$, corresponds to an isothermal sphere; $w=-1, b=3$, to a homogeneous sphere; $w=0, b=0$, to a Roche sphere.

The quintessence mass, $M_{q}(r)$, enclosed by a homogeneous spherical overdensity with equal density and radius as in the infinitely thin sperical shell under consideration, scales as:

$$
\frac{M_{q}(r)}{M_{q}\left(r_{\max }\right)}=\left(\frac{r}{r_{\max }}\right)^{-3 w}
$$

which makes the mass increase as the radius increases, and vice versa.

The validity of Newton's theorem and McLaurin's theorem being independent of the value of the gravitation constant, $G$, homogeneous overdensities where the quintessence is fully clustered evolve in the same way as within static quintessence density profiles, defined by Eqs. (57) or (58), even if the value of the interaction strength is different. In this view, $M_{q}(r)$ is the quintessence mass related to the static profile, enclosed within the volume of the homogeneous overdensity of radius, $r$.

Owing to the above mentioned theorems, (i) given a quintessence spherical corona, related to the static profile, the gravitational action on a selected point enclosed by the inner surface of the corona, is null and (ii) given a quintessence sphere related to the static profile, enclosing a mass, $M_{q}\left(r^{\prime}\right)$, the gravitational action on a selected point outside the surface is equivalent to its counterpart exerted by a central point of equal mass, $M_{q}\left(r^{\prime}\right)$.

Using the above results, the evolution of overdensity infinitely thin, shperical shells may be determined in the limit of fully clustered quintessence. To this aim, the gravitational potential induced by the quintessence is conceived as arising from a distribution where any two "particles", idealized as 
"mass points", $m_{q i}$ and $m_{q j}$, interact with strength, $(1+3 w) G$, according to a Newton-like law, $F_{i j}=(1+3 w) G m_{q i} m_{q j} / r_{i j}$. Then the results related to two-component matter distributions (e.g., Limber, 1959; Brosche et al., 1983; Caimmi et al., 1984; Caimmi and Secco, 1992) may be generalized to the case, where a subsystem is made of quintessence. For further details, refer to Caimmi (2007).

In the special case under consideration $(w=$ const, $\Gamma=0)$ the expansion of an infinitely thin spherical shell enclosing a matter mass, $M_{m}(r)$, and a quintessence mass, $M_{q}(r)$, within a radius, $r$, is expressed as (e.g., Mota and van de Bruck, 2004):

$$
\ddot{r}+\frac{G M_{m}(r)}{r^{2}}+\frac{(1+3 w) G M_{q}(r)}{r^{2}}=0 ;
$$

where mass conservation holds for matter, $M_{\mathrm{m}}(r)=M_{m}\left(r_{i}\right)$, and quintessence mass changes in time according to Eq. (58). The special cases, $w=0$ and $w=-1 / 3$, relate to flat $\left(\Omega_{\mathrm{CDM}}=\Omega_{m}+\Omega_{q}=1\right)$ and open $\left(\Omega_{\mathrm{CDM}}=\Omega_{m}=\right.$ $\left.1-\Omega_{q}\right)$ CDM universes.

The quintessence mass, $M_{q}(r)$, is assumed to change in time according to Eq. (58), which is related to the quintessence mass enclosed by a homogeneous spherical overdensity with equal density and radius as in the infinitely thin shell under consideration. The above approximation overstimates the collapse rate, where the larger effect corresponds to the lower quintessence equation of state parameter, and vice versa. In any case, the effect of the dark energy is negligible in early times (e.g., Weinberg and Kamionkowski, 2003; Horellou and Berge, 2005; Nunes and Mota, 2006), when central collapse is completed.

Following a similar procedure as in presence of sole matter (e.g., Caimmi, 1989), an integration of Eq. (59) yields:

$$
\begin{aligned}
& \dot{r}=\mp\left\{{\dot{r_{i}}}^{2}-\frac{2 G M_{m}\left(r_{i}\right)}{r_{i}}+\frac{2 G M_{m}\left(r_{i}\right)}{r}\right. \\
& \left.-\frac{2(1+3 w) G M_{q}\left(r_{\max }\right)\left(r_{\max }\right)^{3 w}}{r_{i}^{1+3 w}}+\frac{2(1+3 w) G M_{q}\left(r_{\max }\right)\left(r_{\max }\right)^{3 w}}{r^{1+3 w}}\right\}^{1 / 2} ;(60
\end{aligned}
$$

where the plus is related to expansion, and the minus to contraction.

To a good extent, at recombination epoch overdensities still expand together with the universe, which implies the boundary conditions:

$$
r_{i}=r_{h i} ; \quad \dot{r}_{i}=\dot{r}_{h i} ; \quad r_{h i}=a_{h i} r_{h 0} ;
$$


where $r_{h}$ is the radius of a sphere with zero overdensity.

The particularization of Eq. (48) to $a_{h}=a_{h i}$, after combination with Eqs. (19), (33), (46), (47), (58), and (61) yields:

$$
\dot{r}_{i}=\left[\frac{2 G M_{m}\left(r_{i}\right)}{\left(1+\bar{\delta}_{i}\right) r_{i}}+\frac{2 G M_{q}\left(r_{\max }\right)\left(r_{\max }\right)^{3 w}}{r_{i}^{1+3 w}}\right]^{1 / 2} ;
$$

provided isotropic radial motions are dominant.

At turnaround, $\dot{r}=0$ by definition, which implies by use of Eq. (60) particularized to $r=r_{\max }$ :

$$
\begin{aligned}
& \dot{r}_{i}^{2}-\frac{2 G M_{m}\left(r_{i}\right)}{r_{i}}-\frac{2(1+3 w) G M_{q}\left(r_{\max }\right)\left(r_{\max }\right)^{3 w}}{r_{i}^{1+3 w}} \\
& =-\frac{2 G M_{m}\left(r_{i}\right)}{r_{\max }}-\frac{2(1+3 w) G M_{q}\left(r_{\max }\right)}{r_{\max }} ;
\end{aligned}
$$

and the substitution of $\dot{r}_{i}^{2}$ into the general expression, Eq. (60), yields:

$$
\begin{aligned}
& \dot{r}=\mp\left[\frac{2 G M_{m}\left(r_{i}\right)}{r}-\frac{2 G M_{m}\left(r_{i}\right)}{r_{\max }}\right. \\
& \left.+\frac{2(1+3 w) G M_{q}\left(r_{\max }\right)\left(r_{\max }\right)^{3 w}}{r^{1+3 w}}-\frac{2(1+3 w) G M_{q}\left(r_{\max }\right)}{r_{\max }}\right]^{1 / 2} ;
\end{aligned}
$$

where the turnaround radius, $r_{\max }$, has replaced the recombination radius, $r_{i}$.

Using the dimensionless variables:

$$
\begin{aligned}
& \alpha=\frac{r}{r_{\max }} ; \quad \tau=\frac{t}{t_{\max }^{\dagger}} ; \\
& t_{\max }^{\dagger}=\left[\frac{8 G M_{m}\left(r_{i}\right)}{\pi^{2}\left(r_{\max }\right)^{3}}\right]^{-1 / 2}=\left[\frac{32}{3 \pi} G \bar{\rho}\left(r_{\max }\right)\right]^{-1 / 2}
\end{aligned}
$$

together with the fractional mass:

$$
\begin{aligned}
& m=m\left(r_{\max }\right)=\frac{M_{q}\left(r_{\max }\right)}{M_{m}\left(r_{i}\right)} ; \\
& \frac{1}{1+m}=\frac{M_{m}\left(r_{i}\right)}{M_{\mathrm{tot}}\left(r_{\max }\right)} ; \frac{m}{1+m}=\frac{M_{q}\left(r_{\max }\right)}{M_{\mathrm{tot}}\left(r_{\max }\right)} ; M_{\mathrm{tot}}=M_{m}+M_{q} ;
\end{aligned}
$$


allow to cast Eq. (64) under the equivalent form:

$$
\frac{\mathrm{d} \alpha}{\mathrm{d} \tau}=\mp \frac{\pi}{2}\left[\left(\frac{1}{\alpha}-1\right)+(1+3 w) m\left(\frac{1}{\alpha^{1+3 w}}-1\right)\right]^{1 / 2} ;
$$

where the special cases, $w=0$ and $w=-1 / 3$, reproduce the results related to flat $\left(\Omega_{\mathrm{CDM}}=\Omega_{m}+\Omega_{q}=1\right)$ and open $\left(\Omega_{\mathrm{CDM}}=\Omega_{m}=1-\Omega_{q}\right) \mathrm{CDM}$ universes (e.g., Ryden and Gunn, 1987; Caimmi, 1989).

It is worth noticing the scaling time, $t_{\text {max }}^{\dagger}$, defined by Eq. (65b), coincides with the actual matter overdensity free-fall time only for CDM universes i.e. $M_{\text {tot }}\left(r_{\max }\right)=M_{m}\left(r_{\max }\right), M_{q}\left(r_{\max }\right)=0$. In the case under discussion, overdensity collapse is equivalent to pure matter collapse within a static quintessence density profile, $\rho_{q}(r) / \rho_{q}\left(r_{\max }\right)=\left(r / r_{\max }\right)^{-3(1+w)}$, according to Eq. (57). The related free-fall time, to be calculated numerically, is the overdensity free-fall time. Then the dimensionless time, $\tau$, is different from unity at turnaround, unless CDM universes are considered (e.g., Ryden and Gunn, 1987; Caimmi, 1989). In any case, CDM models make a useful reference case, and for this reason the main results shall be recalled in the following.

In the special case, $w=0$, the effect of quintessence is equivalent to the effect of additional matter, $\Omega_{\mathrm{CDM}}=\Omega_{m}+\Omega_{q}=1$, and after a re-definition of the dimensionless time, Eq. (67) reduces to:

$$
\begin{aligned}
& \frac{\mathrm{d} \alpha}{\mathrm{d} \tau}=\mp \frac{\pi}{2} \frac{\sqrt{\alpha-\alpha^{2}}}{\alpha} ; \\
& \tau=\sqrt{1+m} \frac{t}{t_{\max }^{\dagger}} ;
\end{aligned}
$$

where the scaling time, $t_{\max }^{\dagger} / \sqrt{1+m}$, represents the overdensity free-fall time with respect to the turnaround configuration. An integration yields (e.g., Caimmi, 1989):

$$
\tau-\tau_{i}=\mp \frac{2}{\pi}\left[-\sqrt{\alpha-\alpha^{2}}+\sqrt{\alpha_{i}-\alpha_{i}^{2}}-\arcsin \sqrt{1-\alpha}+\arcsin \sqrt{1-\alpha_{i}}\right] \text {; }
$$

in the idealized situation where radial motion is preserved, $\alpha=1$ and $\tau=$ $2 i-1$ at $i$-th turnaround; $\alpha=0$ and $\tau=2 i$ at $i$-th point-like configuration; and Eq. (69) can be splitted as:

$$
\tau=(2 i-1)-\frac{2}{\pi}\left[\sqrt{\alpha-\alpha^{2}}+\arcsin \sqrt{1-\alpha}\right] ;
$$




$$
\tau=(2 i-1)+\frac{2}{\pi}\left[\sqrt{\alpha-\alpha^{2}}+\arcsin \sqrt{1-\alpha}\right] ;
$$

which describe $i$-th expansion and $i$-th contraction, respectively.

For sake of completeness, it is worth recalling the parametric equations (e.g., Ryden and Gunn, 1987; Caimmi, 1989):

$$
\begin{array}{lrl}
\tau=\frac{\theta-\sin \theta}{\pi} ; & \alpha=\frac{1-\cos \theta}{2} ; \\
\frac{\mathrm{d} \tau}{\mathrm{d} \theta}=\frac{2}{\pi} \alpha ; & \frac{\mathrm{d} \alpha}{\mathrm{d} \theta}=\mp \sqrt{\alpha-\alpha^{2}}
\end{array}
$$

where Eq. (68) has been used for the expression of $\mathrm{d} \alpha / \mathrm{d} \theta$.

In the special case, $w=-1 / 3$, the effect of quintessence is equivalent to the effect of additional curvature, $\Omega_{\mathrm{CDM}}=\Omega_{m}=1-\Omega_{q}$, and Eq. (67) reduces to:

$$
\frac{\mathrm{d} \alpha}{\mathrm{d} \tau}=\mp \frac{\pi}{2} \frac{\sqrt{\alpha-\alpha^{2}}}{\alpha}
$$

which coincides with Eq. (68a), and the validity of Eqs. (69)-(71) is maintained.

In the special case, $w=-1$, the effect of quintessence is equivalent to the effect of a cosmological constant, $\Lambda /\left(3 H^{2}\right)=\Omega_{q}$, and Eq. (67) reduces to:

$$
\frac{\mathrm{d} \alpha}{\mathrm{d} \tau}=\mp \frac{\pi}{2}\left[\left(\frac{1}{\alpha}-1\right)-2 m\left(\alpha^{2}-1\right)\right]^{1 / 2} ;
$$

which has no analytical solution and must be solved via numerical integration.

\subsection{Overdensity expansion and central collapse: a spe- cial case}

To the aim of the current attempt, only the early phase of overdensity evolution is considered, from recombination to the end of central collapse, which coincides with the onset of shell crossing in the limit of classical mechanics, where black hole formation does not occur and radial motions are reversed off from the centre. The central collapse may safely be expected to end in a considerably short time, even for massive overdensities. Accordingly, the quintessence effect may be neglected to a first extent. 
From this point on, further effort shall be devoted to the special case, $w=-1 / 3$, for the following reasons: (i) the overdensity equation of motion can analytically be integrated, according to Eq. (69), even in presence of (fully clustered) quintessence; (ii) the (matter + quintessence) overdensity behaves as a pure matter overdensity within an open CDM universe where $\Omega_{\mathrm{CDM}}=$ $\Omega_{m}=1-\Omega_{q}$ (e.g., Horellou and Berge, 2005), and the related results may be extended to the case under discussion; (iii) overdensities reach turnaround and collapse earlier for increasing values of the quintessence equation of state parameter, $w$, and vice versa (e.g., Horellou and Berge, 2005), which makes the case under discussion, $w=-1 / 3$, a lower limit with regard to the range of interest, $-1 \leq w \leq-1 / 3$, where the maximum difference does not exceed about $15 \%$ if collapse ends at present for $w=-1$ (Horellou and Berge, 2005). Concerning central collapse, the above difference is expected to be higly reduced.

According to Eq. (72), at turnaround $\alpha=1$, and the scaling time, $t_{\max }^{\dagger}$, coincides with the free-fall time of the matter subsystem, which implies $\tau_{\max }=1$. For the initial configuration, the combination of Eqs. (42), (65a), and (70a) yields:

$$
\begin{aligned}
& \alpha_{i}=\Delta_{i}=1-\left(\Omega_{m}\right)_{i}^{-1}\left(1+\bar{\delta}_{i}\right)^{-1} \approx \frac{\bar{\delta}_{i}}{1+\bar{\delta}_{i}} ;\left|1-\left(\Omega_{m}\right)_{i}^{-1}\right| \ll \bar{\delta}_{i} \ll 1 ;(74 \mathrm{a}) \\
& \tau_{i}=1-\frac{2}{\pi}\left[\sqrt{\Delta_{i}-\Delta_{i}^{2}}-\arcsin \sqrt{1-\Delta_{i}}\right] .
\end{aligned}
$$

Using the definition of mean density and matter mass conservation, the free-fall time, $t_{\max }$, via Eq. (65b) takes the expression:

$$
t_{\max }=t_{\max }^{\dagger}=\left[\frac{32}{3 \pi} G\left(\bar{\rho}_{m}\right)_{i}\right]^{-1 / 2} \alpha_{i}^{-3 / 2}
$$

where $\left(\bar{\rho}_{m}\right)_{i}$ is the mean density within a sphere of radius, $r_{i}$, centered on a local maximum at recombination epoch. The combination of Eqs. (17), (74a), and (75) yields:

$$
t_{\max }\left(r_{i}\right)=\left[\frac{32}{3 \pi} G\left(\rho_{h m}\right)_{i}\right]^{-1 / 2} \frac{1+\bar{\delta}_{i}\left(r_{i}\right)}{\left[\Delta_{i}\left(r_{i}\right)\right]^{3 / 2}}
$$

where $\left(\rho_{h m}\right)_{i}$ is the density of the matter Hubble flow at recombination epoch. 
The mean overdensity, $\bar{\delta}$, can be related to the initial value, $\bar{\delta}_{i}$, using mass conservation together with Eqs. (19), (70), and (73). The result is:

$$
\begin{aligned}
& \frac{1+\bar{\delta}}{1+\bar{\delta}_{i}}=\left(\frac{\alpha}{\alpha_{h}}\right)^{3} ; \\
& \alpha_{h}=\frac{r_{h}}{r_{\max }} ;
\end{aligned}
$$

where $r_{h}$ is the radius of a sphere with zero overdensity.

Starting from the definition of local density within an infinitely thin spherical shell, and mean density within the volume enclosed by the shell:

$$
\begin{aligned}
\rho_{m}(r) & =\frac{3}{4 \pi r^{2}} \frac{\mathrm{d} M_{m}}{\mathrm{~d} r} ; \\
\bar{\rho}_{m}(r) & =\frac{3}{4 \pi} \frac{M_{m}(r)}{r^{3}} ;
\end{aligned}
$$

mass conservation within the shell and the volume bounded by the shell, with regard to the initial configuration, reads (e.g., Peebles, 1980, Chap. II, $\S 19$ C; Ryden and Gunn, 1987; Caimmi, 1990):

$$
\begin{aligned}
& \rho_{m}(r)=\rho_{m}\left(r_{i}\right)\left(\frac{r}{r_{i}}\right)^{-2}\left(\frac{\partial r}{\partial r_{i}}\right)^{-1} ; \\
& \bar{\rho}_{m}(r)=\bar{\rho}_{m}\left(r_{i}\right)\left(\frac{r}{r_{i}}\right)^{-3} ;
\end{aligned}
$$

and the combination of Eqs. (19) and (77)-(81) yields:

$$
\begin{aligned}
& \frac{\rho_{m}(r)}{\bar{\rho}_{m}(r)}=\frac{1+\delta(r)}{1+\bar{\delta}(r)}=\frac{1+\delta_{i}\left(r_{i}\right)}{1+\bar{\delta}_{i}\left(r_{i}\right)} g(r) ; \\
& g(r)=\frac{r}{r_{i}}\left(\frac{\partial r}{\partial r_{i}}\right)^{-1} ;
\end{aligned}
$$

where the mean overdensity, $\bar{\delta}(r)$, can be determined for a fixed cosmological model via Eqs. (69) and (77), and the explicit expression of the partial derivative, $\partial r / \partial r_{i}$, allows the explicit expression of the local overdensity, $\delta(r)$, via Eqs. (77).

The related procedure, involving long but stimulating algebra, has been performed in different situations related to CDM cosmologies: overdensity 
evolution in Einstein-de Sitter universes (Peebles, 1980, Chap. II, § 19C; Ryden and Gunn, 1987; Caimmi, 1989); overdensity evolution in Einstein-de Sitter universes with the effects of acquisition of angular momentum on the expansion included (Caimmi, 1990); overdensity evolution in high-energy $\left(\Omega_{m} \gtrsim 0.1\right)$ universes (Andriani and Caimmi, 1991); overdensity evolution in high-energy $\left(\Omega_{m} \gtrsim 0.1\right)$ universes with the effects of acquisition of angular momentum on the expansion included (Andriani and Caimmi, 1994). The third above mentioned case (Andriani and Caimmi, 1991) shall be considered in the current attempt.

Accordingly, the explicit expression of Eq. (82b) reads:

$$
\begin{aligned}
& {[g(r)]^{-1}=1+3 \frac{1-\Delta_{i}}{\Delta_{i}} \frac{\bar{\delta}_{i}}{1+\bar{\delta}_{i}}\left(1-\frac{\delta_{i}}{\bar{\delta}_{i}}\right)\left\{1-\frac{\Delta_{i}^{2}}{\sqrt{\Delta_{i}-\Delta_{i}^{2}}} \frac{\mp \sqrt{\alpha-\alpha^{2}}}{\alpha^{2}} \times\right.} \\
& \left.\times\left[1+\frac{3 \pi}{4}\left(1+\bar{\delta}_{i}\right)^{1 / 2}\left(\frac{1-\Delta_{i}}{\Delta_{i}^{3}}\right)^{1 / 2}\left(1+\frac{1}{3} \frac{\Delta_{i}}{1-\Delta_{i}}\right)\left(\tau-\tau_{i}\right)\right]\right\} ;
\end{aligned}
$$

where the positive and the negative sign within parentheses are related to shell expansion and contraction, respectively. For a formal derivation, see Appendix C.

For density profiles obeying Eq. (5), overdensity central collapse is completed at a dimensionless time, $\tau\left(r_{\mathrm{pk}}\right)=2$, according to Eq. (70b), where $r_{\mathrm{pk}}$ is the radius of the homogeneous peak at recombination epoch.

Let $\tau_{c}\left(r_{i}\right)$ be the dimensionless time related to an infinitely thin spherical shell of initial radius, $r_{i}$, at the end of central collapse i.e. $t=2 t_{\max }\left(r_{\mathrm{pk}}\right)$. The combination of Eqs. (65) and (76) produces:

$$
\tau_{c}\left(r_{i}\right)=2 \frac{1+\delta_{\mathrm{pk}}}{1+\bar{\delta}_{i}\left(r_{i}\right)}\left[\frac{\bar{\delta}_{i}\left(r_{i}\right)}{\delta_{\mathrm{pk}}}\right]^{3 / 2} ;
$$

where $\delta_{\mathrm{pk}}=\bar{\delta}_{i}\left(r_{\mathrm{pk}}\right)$ is the overdensity value of the homogeneous peak, and $\bar{\delta}_{i}\left(r_{i}\right)$ is related to the initial density profile via Eqs. (23) and (27).

The related dimensionless distance, $\alpha_{c}\left(r_{i}\right)$, can be determined as the solution to the transcendental equation deduced from Eq. (70b):

$$
\tau_{c}\left(r_{i}\right)=1+\frac{2}{\pi}\left[\sqrt{\alpha_{c}\left(r_{i}\right)-\alpha_{c}^{2}\left(r_{i}\right)}+\arcsin \sqrt{1-\alpha_{c}\left(r_{i}\right)}\right] ;
$$


and the combination of Eqs. (65a) and (74a) yields:

$$
\alpha_{c}\left(r_{i}\right)=\frac{r_{c}\left(r_{i}\right)}{r_{i}} \frac{r_{i}}{r_{\max }\left(r_{i}\right)}=\frac{r_{c}\left(r_{i}\right)}{r_{i}} \alpha_{i}\left(r_{i}\right)=\frac{r_{c}\left(r_{i}\right)}{r_{i}} \Delta_{i}\left(r_{i}\right) ;
$$

which allows an explicit expression of the radius of an infinitely thin spherical shell at the end of central collapse, as:

$$
r_{c}\left(r_{i}\right)=\frac{\alpha_{c}\left(r_{i}\right) r_{i}}{\Delta_{i}\left(r_{i}\right)}
$$

where $r_{i}$ is the initial radius at recombination epoch.

The density profile at the end of central collapse, follows from the particularization of Eqs. (82) and (83) to the case of interest. The result is:

$$
\begin{aligned}
& \frac{\rho\left(r_{c}\right)}{\bar{\rho}\left(r_{c}\right)}=\frac{1+}{1+} \bar{\delta}\left(r_{c}\right) \\
& {\left[g\left(r_{c}\right)\right]^{-1}=} \frac{1+\delta_{i}\left(r_{i}\right)}{1+\bar{\delta}_{i}\left(r_{i}\right)} g\left(r_{c}\right) \\
&+3 \frac{1-\Delta_{i}\left(r_{i}\right)}{\Delta_{i}\left(r_{i}\right)} \frac{\bar{\delta}_{i}\left(r_{i}\right)}{1+\bar{\delta}_{i}\left(r_{i}\right)}\left[1-\frac{\delta_{i}\left(r_{i}\right)}{\bar{\delta}_{i}\left(r_{i}\right)}\right]\left\{1-\frac{\left[\Delta_{i}\left(r_{i}\right)\right]^{2}}{\sqrt{\Delta_{i}\left(r_{i}\right)-\left[\Delta_{i}\left(r_{i}\right)\right]^{2}}}\right. \\
& \times \frac{\mp \sqrt{\alpha_{c}\left(r_{i}\right)-\left[\alpha_{c}\left(r_{i}\right)\right]^{2}}}{\left[\alpha_{c}\left(r_{i}\right)\right]^{2}}\left[1+\frac{3 \pi}{4}\left[1+\bar{\delta}_{i}\left(r_{i}\right)\right]^{1 / 2}\left[\frac{1-\Delta_{i}\left(r_{i}\right)}{\Delta_{i}^{3}\left(r_{i}\right)}\right]^{1 / 2}\right. \\
&\left.\left.\times\left[1+\frac{1}{3} \frac{\Delta_{i}\left(r_{i}\right)}{1-\Delta_{i}\left(r_{i}\right)}\right]\left[\tau_{c}\left(r_{i}\right)-\tau_{i}\left(r_{i}\right)\right]\right]\right\}
\end{aligned}
$$

where global and local overdensities, $\bar{\delta}_{i}$ and $\delta_{i}$, are determined by the knowledge of the initial density profile at recombination epoch, via Eqs. (23) and (24), respectively.

The overdensity evolution obeying an initial density profile defined by Eq. (5), lies between the limiting situations, $b=0$ and $b=3$. The special case, $b=0$, corresponds to a Roche sphere i.e. a mass point surrounded by a massless atmosphere. Accordingly, $r_{\mathrm{pk}}=0$ and the hole is present from the beginning of evolution. The special case, $b=3$, corresponds to a homogeneous sphere which collapses into a hole after a time, $t=2 t_{\max }\left(R_{i}\right)$. In the general case, $0<b<3$, the homogeneous peak collapses into a hole after a time, $t=2 t_{\max }\left(r_{\mathrm{pk}}\right)$, while the underlying envelope virializes due to deviations from radial motion. 
According to the results of Subsect. 2.3, the dimensionless radius, $\Xi=$ $R / r_{\mathrm{pk}}$, Eq. (8), and the dimensionless densities, $\bar{\rho}_{\mathrm{tr}} / \rho_{\mathrm{pk}}$ and $\rho_{\mathrm{tr}} / \rho_{\mathrm{pk}}$, Eqs. (10) and (11), respectively, depend only on the input parameters, $b$ and $\kappa$. For changing masses and peak heights, the above mentioned quantities necessarily remain fixed. With regard to the mass ratio, $\kappa=M_{\mathrm{pk}} / M_{\mathrm{tr}}$, an empirical relation between hole and hosting spheroid mass (e.g., Ferrarese and Ford, 2005) cannot be used for for the following reasons.

First, the above mentioned relation holds for ellipticals and early-type spirals, but the disk mass is not taken into consideration. On the other hand, the disk is dominant in late-type spirals, and both the proto-bulge and the proto-disk, together with the (nonbaryonic) dark matter, must be included in the initial density profile at recombination epoch.

Second, the dark matter is dominant with respect to the baryonic matter, and the relation between dark matter and hole mass seems to be nonlinear, as:

$$
\frac{M_{\text {hole }}}{M_{\text {dark }}}=5.810^{-5}\left(\frac{1}{100} \frac{M_{\text {dark }}}{\mathrm{M}_{10}}\right)^{\beta} \frac{1+z}{7} ; \quad 10 \lesssim M_{\text {dark }} / \mathrm{M}_{10} \lesssim 1000 ;
$$

where $z$ is the redshift and $\beta$ an exponent to be fixed. Special values are: $(\beta, z)=(0.65,6 / 29)$ (Ferrarese, 2002); $(\beta, z)=(0.27,19 / 58)$ (Baes et al., 2003); and $\beta=0.39$ (Shankar and Mathur, 2007). In addition, Eq. (89) is close to its counterpart calibrated locally through statistical arguments (Shankar et al., 2006) and to its counterparts obtained using semianalytical models (Granato et al., 2004; Lapi et al., 2006). It can be seen that a redshift-dependent superlinear relation, $M_{\text {hole }} / M_{\text {dark }} \propto M_{\text {dark }}^{\beta}, 0.3 \lesssim \beta \lesssim 0.7$, is best suited to represent the luminosity function in active galactic nuclei (Shankar et al., 2007). Accordingly, Eq. (89) shall be used in computations. An extrapolation to the mass range under consideration, $10^{-1} \leq M / \mathrm{M}_{10} \leq$ $10^{6}$, via Eq. (89) yields $10^{-8}<M_{\text {hole }} / \mathrm{M}_{10}<10^{4}$ in the special case, $\beta=0.65$, $z=6 / 29$.

If the lack of low-mass $\left(10^{-7} \lesssim M_{\text {hole }} / \mathrm{M}_{10} \lesssim 10^{-4}\right)$ holes is real, a possible interpretation could be the following. Secondary perturbations naturally arise around a local density maximum (e.g., Ryden and Gunn, 1987) and virialize when the hosting overdensity is still expanding. For total clump number or typical clump mass independent of overdensity mass, it can be seen that the distance between nearest clumps (provided they are uniformly distributed within the overdensity volume) increases as the overdensity mass 
increases, and vice versa. Accordingly, tidal interactions and related departure from radial motions are expected to be more efficient in low-mass $\left(M \lesssim 10^{2} \mathrm{M}_{10}\right.$ ) overdensities, which could prevent hole formation.

\section{Results}

Computations have been performed up to the end of the central collapse, within the mass range, $-1 \leq \log \left(M / \mathrm{M}_{10}\right) \leq 6$, for mean overdensity heights, $\bar{\nu}=1,2,3,4$, and an assumed quintessence equation of state parameter, $w=$ $-1 / 3$. The density profile at the end of central collapse, and related values of dimensionless times, $\tau_{c}\left(r_{i}\right)$, and dimensionless radii, $\alpha_{c}\left(r_{i}\right)$, are weakly dependent on the mean overdensity height, to a major extent as the initial shell radius, $r_{i}$, is selected near the truncation radius. In addition, different overdensity heights related to low masses exhibit slightly larger differences with respect to their counterparts related to high masses. Also, differences in $\tau_{c}$ and $\alpha_{c}$ are more pronounced (but still negligible) in comparison to differences in density, provided the remaining parameters are kept fixed.

The ratio of homogeneous peak radius to truncation radius, $\Xi^{-1}=r_{\mathrm{pk}} / R_{\mathrm{tr}}$, mean density to homogeneous peak density, $\bar{\rho}_{\mathrm{tr}} / \rho_{\mathrm{pk}}$, and local density at truncation radius to homogeneous peak density, $\rho_{\mathrm{tr}} / \rho_{\mathrm{pk}}$, depend only on the slope of the external density profile, $b$, and the ratio of homogeneous peak mass to total mass within the truncation radius, $\kappa=M_{\mathrm{pk}} / M_{\mathrm{tr}}$, according to Eqs. (8), (10), and (11), respectively. The dependence within the ranges, $2.85 \leq b \leq 3,-8 \leq \log \kappa \leq-2$, is shown in Tab. 2. The dependence on the power-law density profile exponent, $b$, for fixed ratio of homogeneous peak mass to total mass within the truncation radius, $\kappa$, is steeper for low $\kappa$ and vice versa.

For proto-galaxies $\left(M / \mathrm{M}_{10}=100\right)$, slopes $b<2.85$ would imply exceedingly large initial peak overdensities $\left(\delta_{\mathrm{pk}}>0.74\right)$, and slopes $b>2.996$ would imply exceedingly large cosmic times at the end of central collapse $\left(t_{c}>0.9 \mathrm{Gyr}\right)$. Mass ratios, $\kappa$, outside the above mentioned range would be inconsistent with Eq. (89), related to the cases of interest deduced from observations and/or supported by theoretical arguments. The homogeneous density profile, $b=3$, makes a useful limiting case.

In the current model, the hole mass coincides with the homogeneous peak mass, and $\kappa$ can be deduced from Eq. (89). As a reference case, the values 


\begin{tabular}{lcccccc}
\hline \hline $\log \kappa=-8$ & -7 & -6 & -5 & -4 & -3 & -2 \\
\hline $1.588 \mathrm{E}-3$ & $3.562 \mathrm{E}-3$ & $7.990 \mathrm{E}-3$ & $1.792 \mathrm{E}-2$ & $4.021 \mathrm{E}-2$ & $9.019 \mathrm{E}-2$ & $2.023 \mathrm{E}-1$ \\
$4.003 \mathrm{E}-1$ & $4.519 \mathrm{E}-1$ & $5.101 \mathrm{E}-1$ & $5.758 \mathrm{E}-1$ & $6.500 \mathrm{E}-1$ & $7.337 \mathrm{E}-1$ & $8.278 \mathrm{E}-1$ \\
$3.803 \mathrm{E}-1$ & $4.293 \mathrm{E}-1$ & $4.846 \mathrm{E}-1$ & $5.470 \mathrm{E}-1$ & $6.175 \mathrm{E}-1$ & $6.971 \mathrm{E}-1$ & $7.869 \mathrm{E}-1$ \\
$1.530 \mathrm{E}-0$ & $1.241 \mathrm{E}-0$ & $9.823 \mathrm{E}-1$ & $7.587 \mathrm{E}-1$ & $5.580 \mathrm{E}-1$ & $3.802 \mathrm{E}-1$ & $2.233 \mathrm{E}-1$ \\
& & & & & & \\
$1.764 \mathrm{E}-3$ & $3.902 \mathrm{E}-3$ & $8.632 \mathrm{E}-3$ & $1.910 \mathrm{E}-2$ & $4.224 \mathrm{E}-2$ & $9.345 \mathrm{E}-2$ & $2.067 \mathrm{E}-1$ \\
$5.487 \mathrm{E}-1$ & $5.941 \mathrm{E}-1$ & $6.432 \mathrm{E}-1$ & $6.963 \mathrm{E}-1$ & $7.539 \mathrm{E}-1$ & $8.161 \mathrm{E}-1$ & $8.833 \mathrm{E}-1$ \\
$5.304 \mathrm{E}-1$ & $5.743 \mathrm{E}-1$ & $6.217 \mathrm{E}-1$ & $6.731 \mathrm{E}-1$ & $7.287 \mathrm{E}-1$ & $7.890 \mathrm{E}-1$ & $8.542 \mathrm{E}-1$ \\
$8.455 \mathrm{E}-1$ & $7.046 \mathrm{E}-1$ & $5.745 \mathrm{E}-1$ & $4.543 \mathrm{E}-1$ & $3.433 \mathrm{E}-1$ & $2.408 \mathrm{E}-1$ & $1.465 \mathrm{E}-1$ \\
& & & & & & \\
$1.953 \mathrm{E}-3$ & $4.262 \mathrm{E}-3$ & $9.302 \mathrm{E}-3$ & $2.030 \mathrm{E}-2$ & $4.431 \mathrm{E}-2$ & $9.672 \mathrm{E}-2$ & $2.111 \mathrm{E}-1$ \\
$7.444 \mathrm{E}-1$ & $7.741 \mathrm{E}-1$ & $8.049 \mathrm{E}-1$ & $8.369 \mathrm{E}-1$ & $8.702 \mathrm{E}-1$ & $9.048 \mathrm{E}-1$ & $9.407 \mathrm{E}-1$ \\
$7.320 \mathrm{E}-1$ & $7.612 \mathrm{E}-1$ & $7.915 \mathrm{E}-1$ & $8.230 \mathrm{E}-1$ & $8.557 \mathrm{E}-1$ & $8.898 \mathrm{E}-1$ & $9.252 \mathrm{E}-1$ \\
$3.603 \mathrm{E}-1$ & $3.083 \mathrm{E}-1$ & $2.582 \mathrm{E}-1$ & $2.100 \mathrm{E}-1$ & $1.637 \mathrm{E}-1$ & $1.192 \mathrm{E}-1$ & $7.654 \mathrm{E}-2$ \\
& & & & & & \\
$2.154 \mathrm{E}-3$ & $4.642 \mathrm{E}-3$ & $1.000 \mathrm{E}-2$ & $2.154 \mathrm{E}-2$ & $4.642 \mathrm{E}-2$ & $1.000 \mathrm{E}-1$ & $2.154 \mathrm{E}-1$ \\
$1.000 \mathrm{E}-0$ & $1.000 \mathrm{E}-0$ & $1.000 \mathrm{E}-0$ & $1.000 \mathrm{E}-0$ & $1.000 \mathrm{E}-0$ & $1.000 \mathrm{E}-0$ & $1.000 \mathrm{E}-0$ \\
$1.000 \mathrm{E}-0$ & $1.000 \mathrm{E}-0$ & $1.000 \mathrm{E}-0$ & $1.000 \mathrm{E}-0$ & $1.000 \mathrm{E}-0$ & $1.000 \mathrm{E}-0$ & $1.000 \mathrm{E}-0$ \\
$1.270 \mathrm{E}-2$ & $1.270 \mathrm{E}-2$ & $1.270 \mathrm{E}-2$ & $1.270 \mathrm{E}-2$ & $1.270 \mathrm{E}-2$ & $1.270 \mathrm{E}-2$ & $1.270 \mathrm{E}-2$ \\
\hline \hline
\end{tabular}

Table 2: From up to down of each block: ratio of homogeneous peak radius to truncation radius, $\Xi^{-1}=r_{\mathrm{pk}} / R_{\mathrm{tr}}$ (first lines), mean density to homogeneous peak density, $\bar{\rho}_{\mathrm{tr}} / \rho_{\mathrm{pk}}$ (second lines), local density at truncation radius to homogeneous peak density, $\rho_{\mathrm{tr}} / \rho_{\mathrm{pk}}$ (third lines), and peak overdensity, $\delta_{\mathrm{pk}}$, (fourth lines), for different choices of ratio of homogeneous peak mass to total mass within the truncation radius, $\kappa=M_{\mathrm{pk}} / M_{\mathrm{tr}}$, and power-law density profile exponent (from top to bottom), $b=2.85$ (first block); $b=2.90$ (second block); $b=2.95$ (third block); $b=3$ (fourth block). 


\begin{tabular}{cccccc}
\hline \hline $\log \left(M / \mathrm{M}_{10}\right)$ & $R / \mathrm{kpc}$ & $r_{\mathrm{pk}} / \mathrm{kpc}$ & $\bar{\delta}_{\mathrm{M}}$ & $\kappa$ & $\Xi^{-1}$ \\
\hline-1 & $1.72 \mathrm{E}-1$ & $6.98 \mathrm{E}-4$ & $1.27 \mathrm{E}-2$ & $1.12 \mathrm{E}-7$ & $4.06 \mathrm{E}-3$ \\
0 & $3.70 \mathrm{E}-1$ & $2.52 \mathrm{E}-3$ & $1.00 \mathrm{E}-2$ & $5.01 \mathrm{E}-7$ & $6.80 \mathrm{E}-3$ \\
1 & $7.98 \mathrm{E}-1$ & $9.10 \mathrm{E}-3$ & $7.26 \mathrm{E}-3$ & $2.24 \mathrm{E}-6$ & $1.14 \mathrm{E}-2$ \\
2 & $1.72 \mathrm{E}+0$ & $3.28 \mathrm{E}-2$ & $4.81 \mathrm{E}-3$ & $1.00 \mathrm{E}-5$ & $1.91 \mathrm{E}-2$ \\
3 & $3.70 \mathrm{E}+0$ & $1.18 \mathrm{E}-1$ & $3.08 \mathrm{E}-3$ & $4.47 \mathrm{E}-5$ & $3.20 \mathrm{E}-2$ \\
4 & $7.98 \mathrm{E}+0$ & $4.28 \mathrm{E}-1$ & $1.72 \mathrm{E}-3$ & $1.99 \mathrm{E}-4$ & $5.36 \mathrm{E}-2$ \\
5 & $1.72 \mathrm{E}+1$ & $1.54 \mathrm{E}+0$ & $7.89 \mathrm{E}-4$ & $8.91 \mathrm{E}-4$ & $8.98 \mathrm{E}-2$ \\
6 & $3.70 \mathrm{E}+1$ & $5.57 \mathrm{E}+0$ & $2.90 \mathrm{E}-4$ & $3.98 \mathrm{E}-3$ & $1.50 \mathrm{E}-1$ \\
\hline \hline
\end{tabular}

Table 3: The homogeneous peak radius, $r_{\mathrm{pk}}$, the ratio of homogeneous peak radius to truncation radius, $\Xi^{-1}=r_{\mathrm{pk}} / R_{\mathrm{tr}}$, and the ratio of homogeneous peak mass to total mass within the truncation radius, $\kappa=M_{\mathrm{pk}} / M_{\mathrm{tr}}$, for overdensities with different masses, at recombination epoch, density profile defined by Eq. (5), $\kappa$ deduced from Eq. (89), particularized to the selected reference case, $b=2.90$ and $(\beta, z)=(0.65,6 / 29)$. The rms overdensity value, $\left(\bar{\delta}_{M}\right)_{i}=\bar{\delta}_{M}$, and the overdensity radius, $R_{i}=R$, are taken from Tab. 1 for sake of completeness.

$(\beta, z)=(0.65,6 / 29)$ shall be chosen, which make Eq. (89) coincide with its empirical counterpart deduced from a sample of 37 spiral galaxies (Ferrarese, 2002), together with the value, $b=2.90$ in Eq. (5). The related values of the homogeneous peak radius, $r_{\mathrm{pk}}$, and the ratio of the homogeneous peak radius to truncation radius, $\Xi^{-1}=r_{\mathrm{pk}} / R_{\mathrm{tr}}$, are listed in Tab. 3 for different masses at the beginning of evolution, assumed to be at recombination epoch. Initial radii, $R_{i}=R$, and rms overdensity values, $\left(\bar{\delta}_{\mathrm{M}}\right)_{i}=\bar{\delta}_{\mathrm{M}}$, are taken from Tab. 1 for sake of completeness. The above mentioned quantities are independent of the overdensity height.

The homogeneous peak overdensity, $\delta_{\mathrm{pk}}$, the local overdensity at the truncation radius, $\delta_{\mathrm{tr}}$, the homogeneous peak density, $\rho_{\mathrm{pk}}$, the overdensity turnaround radius, $R_{\max }$, the cosmic time at turnaround, $t_{\max }$, and at the end of central collapse, $t_{c}$, are listed in Tab. 4 for mean overdensity heights, $\bar{\nu}_{i}=1,2,3,4$ (from top to bottom in each bloch), and different masses, with regard to the selected reference case. The homogeneous peak density, $\rho_{\mathrm{pk}}$, and the local overdensity at truncation radius, $\delta_{\text {tr }}$, decrease only slowly with increasing masses, and the same holds for the homogeneous peak overden- 


\begin{tabular}{|c|c|c|c|c|c|c|}
\hline $\log \frac{M}{\mathrm{M}_{10}}$ & $\delta_{\mathrm{pk}}$ & $\delta_{\mathrm{tr}}$ & $\frac{\rho_{\mathrm{pk}}}{\left(\mathrm{M}_{10} / \mathrm{kpc}^{3}\right)}$ & $\frac{r_{\max }}{\mathrm{kpc}}$ & $\frac{t_{\max }}{\text { Gyr }}$ & $\frac{t_{c}}{\mathrm{Gyr}}$ \\
\hline \multirow{4}{*}{-1} & $6.98 \mathrm{E}-1$ & $-2.11 \mathrm{E}-2$ & $7.97 \mathrm{E}+0$ & $1.37 \mathrm{E}+1$ & $8.51 \mathrm{E}-1$ & $1.55 \mathrm{E}-2$ \\
\hline & $7.19 \mathrm{E}-1$ & $-8.78 \mathrm{E}-3$ & $8.07 \mathrm{E}+0$ & $6.54 \mathrm{E}+0$ & $3.05 \mathrm{E}-1$ & $1.53 \mathrm{E}-2$ \\
\hline & $7.40 \mathrm{E}-1$ & $+3.49 \mathrm{E}-3$ & $8.17 \mathrm{E}+0$ & $4.69 \mathrm{E}+0$ & $1.68 \mathrm{E}-1$ & $1.51 \mathrm{E}-2$ \\
\hline & $7.62 \mathrm{E}-1$ & $+1.58 \mathrm{E}-2$ & $8.27 \mathrm{E}+0$ & $3.56 \mathrm{E}+0$ & $1.10 \mathrm{E}-1$ & $1.49 \mathrm{E}-2$ \\
\hline \multirow[t]{4}{*}{0} & $6.08 \mathrm{E}-1$ & $-2.37 \mathrm{E}-2$ & $7.55 \mathrm{E}+0$ & $3.75 \mathrm{E}+1$ & $1.22 \mathrm{E}+0$ & $1.66 \mathrm{E}-2$ \\
\hline & $6.24 \mathrm{E}-1$ & $-1.40 \mathrm{E}-2$ & $7.62 \mathrm{E}+0$ & $1.89 \mathrm{E}+1$ & $4.35 \mathrm{E}-1$ & $1.64 \mathrm{E}-2$ \\
\hline & $6.40 \mathrm{E}-1$ & $-4.40 \mathrm{E}-3$ & $7.70 \mathrm{E}+0$ & $1.27 \mathrm{E}+1$ & $2.39 \mathrm{E}-1$ & $1.62 \mathrm{E}-2$ \\
\hline & $6.56 \mathrm{E}-1$ & $+5.24 \mathrm{E}-3$ & $7.77 \mathrm{E}+0$ & $9.65 \mathrm{E}+0$ & $1.57 \mathrm{E}-1$ & $1.60 \mathrm{E}-2$ \\
\hline \multirow[t]{4}{*}{1} & $5.23 \mathrm{E}-1$ & $-2.63 \mathrm{E}-2$ & $7.15 \mathrm{E}+0$ & $1.11 \mathrm{E}+2$ & $1.96 \mathrm{E}+0$ & $1.82 \mathrm{E}-2$ \\
\hline & $5.34 \mathrm{E}-1$ & $-1.93 \mathrm{E}-2$ & $7.20 \mathrm{E}+0$ & $5.58 \mathrm{E}+1$ & $6.98 \mathrm{E}-1$ & $1.80 \mathrm{E}-2$ \\
\hline & $5.45 \mathrm{E}-1$ & $-1.23 \mathrm{E}-2$ & $7.25 \mathrm{E}+0$ & $3.75 \mathrm{E}+1$ & $3.83 \mathrm{E}-1$ & $1.77 \mathrm{E}-2$ \\
\hline & $5.56 \mathrm{E}-1$ & $-5.28 \mathrm{E}-3$ & $7.31 \mathrm{E}+0$ & $2.83 \mathrm{E}+1$ & $2.50 \mathrm{E}-1$ & $1.75 \mathrm{E}-2$ \\
\hline \multirow[t]{4}{*}{2} & $4.43 \mathrm{E}-1$ & $-2.87 \mathrm{E}-2$ & $6.77 \mathrm{E}+0$ & $3.59 \mathrm{E}+2$ & $3.63 \mathrm{E}+0$ & $2.04 \mathrm{E}-2$ \\
\hline & $4.50 \mathrm{E}-1$ & $-2.40 \mathrm{E}-2$ & $6.81 \mathrm{E}+0$ & $1.81 \mathrm{E}+2$ & $1.29 \mathrm{E}+0$ & $2.02 \mathrm{E}-2$ \\
\hline & $4.57 \mathrm{E}-1$ & $-1.93 \mathrm{E}-2$ & $6.84 \mathrm{E}+0$ & $1.21 \mathrm{E}+2$ & $7.05 \mathrm{E}-1$ & $2.00 \mathrm{E}-2$ \\
\hline & $4.64 \mathrm{E}-1$ & $-1.47 \mathrm{E}-2$ & $6.87 \mathrm{E}+0$ & $9.11 \mathrm{E}+1$ & $4.60 \mathrm{E}-1$ & $1.98 \mathrm{E}-2$ \\
\hline \multirow[t]{4}{*}{3} & $3.68 \mathrm{E}-1$ & $-3.03 \mathrm{E}-2$ & $6.42 \mathrm{E}+0$ & $1.20 \mathrm{E}+3$ & $7.05 \mathrm{E}+0$ & $2.36 \mathrm{E}-2$ \\
\hline & $3.72 \mathrm{E}-1$ & $-2.74 \mathrm{E}-2$ & $6.44 \mathrm{E}+0$ & $6.04 \mathrm{E}+2$ & $2.50 \mathrm{E}+0$ & $2.34 \mathrm{E}-2$ \\
\hline & $3.76 \mathrm{E}-1$ & $-2.44 \mathrm{E}-2$ & $6.46 \mathrm{E}+0$ & $4.04 \mathrm{E}+2$ & $1.36 \mathrm{E}+0$ & $2.32 \mathrm{E}-2$ \\
\hline & $3.81 \mathrm{E}-1$ & $-2.14 \mathrm{E}-2$ & $6.48 \mathrm{E}+0$ & $3.04 \mathrm{E}+2$ & $8.89 \mathrm{E}-1$ & $2.29 \mathrm{E}-2$ \\
\hline \multirow[t]{4}{*}{4} & $2.97 \mathrm{E}-1$ & $-3.17 \mathrm{E}-2$ & $6.09 \mathrm{E}+0$ & $4.64 \mathrm{E}+3$ & $1.68 \mathrm{E}+1$ & $2.84 \mathrm{E}-2$ \\
\hline & $3.00 \mathrm{E}-1$ & $-3.00 \mathrm{E}-2$ & $6.10 \mathrm{E}+0$ & $2.32 \mathrm{E}+3$ & $5.97 \mathrm{E}+0$ & $2.82 \mathrm{E}-2$ \\
\hline & $3.02 \mathrm{E}-1$ & $-2.83 \mathrm{E}-2$ & $6.11 \mathrm{E}+0$ & $1.55 \mathrm{E}+3$ & $3.25 \mathrm{E}+0$ & $2.80 \mathrm{E}-2$ \\
\hline & $3.04 \mathrm{E}-1$ & $-2.67 \mathrm{E}-2$ & $6.12 \mathrm{E}+0$ & $1.17 \mathrm{E}+3$ & $2.12 \mathrm{E}+0$ & $2.79 \mathrm{E}-2$ \\
\hline \multirow[t]{4}{*}{5} & $2.31 \mathrm{E}-1$ & $-3.25 \mathrm{E}-2$ & $5.78 \mathrm{E}+0$ & $2.18 \mathrm{E}+4$ & $5.43 \mathrm{E}+1$ & $3.64 \mathrm{E}-2$ \\
\hline & $2.32 \mathrm{E}-1$ & $-3.18 \mathrm{E}-2$ & $5.78 \mathrm{E}+0$ & $1.09 \mathrm{E}+4$ & $1.92 \mathrm{E}+1$ & $3.63 \mathrm{E}-2$ \\
\hline & $2.33 \mathrm{E}-1$ & $-3.10 \mathrm{E}-2$ & $5.79 \mathrm{E}+0$ & $7.28 \mathrm{E}+3$ & $1.05 \mathrm{E}+1$ & $3.61 \mathrm{E}-2$ \\
\hline & $2.34 \mathrm{E}-1$ & $-3.02 \mathrm{E}-2$ & $5.79 \mathrm{E}+0$ & $5.46 \mathrm{E}+3$ & $6.81 \mathrm{E}+0$ & $3.60 \mathrm{E}-2$ \\
\hline \multirow[t]{4}{*}{6} & $1.69 \mathrm{E}-1$ & $-3.29 \mathrm{E}-2$ & $5.49 \mathrm{E}+0$ & $1.28 \mathrm{E}+5$ & $2.43 \mathrm{E}+2$ & $5.13 \mathrm{E}-2$ \\
\hline & $1.69 \mathrm{E}-1$ & $-3.26 \mathrm{E}-2$ & $5.49 \mathrm{E}+0$ & $6.39 \mathrm{E}+4$ & $8.61 \mathrm{E}+1$ & $5.11 \mathrm{E}-2$ \\
\hline & $1.69 \mathrm{E}-1$ & $-3.24 \mathrm{E}-2$ & $53 \sqrt{3} \mathrm{E}+0$ & $4.26 \mathrm{E}+4$ & $4.69 \mathrm{E}+1$ & $5.10 \mathrm{E}-2$ \\
\hline & $1.70 \mathrm{E}-1$ & $-3.21 \mathrm{E}-2$ & $5.49 \mathrm{E}+0$ & $3.19 \mathrm{E}+4$ & $3.04 \mathrm{E}+1$ & $5.09 \mathrm{E}-2$ \\
\hline
\end{tabular}

Table 4: Homogeneous peak overdensity, $\delta_{\mathrm{pk}}$, local overdensity at truncation radius, $\delta_{\text {tr }}$, homogeneous peak density, $\rho_{\mathrm{pk}}$, overdensity turnaround radius, $R_{\max }$, cosmic time at turnaround, $t_{\max }$, and at the end of central collapse, $t_{c}$, for mean overdensity heights, $\bar{\nu}_{i}=1,2,3,4$ (from top to bottom of each 
sity, $\delta_{\mathrm{pk}}$, though to a slightly larger extent. In particular, the model density profile, expressed by Eq. (5), implies the existence of an underdense external region (negative overdensity) up to the truncation radius, at the end of central collapse. Both the overdensity turnaround radius, $r_{\max }$, and the cosmic time at the end of central collapse, $t_{c}$, decrease for increasing mean overdensity height, $\bar{\nu}_{i}$. Accordingly, in the light of the model under discussion, hole formation occurs within a few hundredths of Gyr, or $z>10$.

The fractional density at the end of central collapse, $\rho / \bar{\rho}$, as a function of the fractional radius, $\xi=r / R$, at the end of central collapse, for different masses and mean overdensity heights, with regard to the selected reference case, is plotted in Fig. 1. The density profile of the outer shells, which are still expanding together with the universe, exhibits no dependence on the overdensity mass. The contrary holds for the inner shells where, for assigned fractional radius, the fractional density is an increasing function of the overdensity mass. The dependence on the mean overdensity height appears to be negligible.

The density, $\rho$, as a function of the radius, $r$, at the end of central collapse, for different masses and mean overdensity heights, with regard to the selected reference case, is plotted on a logarithmic plane in Fig. 2. The density profile is universal, with the exception of the inner shells, where a higher density is attained. The dependence on the mean overdensity height appears to be negligible.

The dimensionless time, $\tau=\tau_{c}\left(r_{i}\right)=t_{c} / t_{\max }\left(r_{i}\right)$, as a function of the fractional radius, $\xi=r / R$, at the end of central collapse, for different masses and mean overdensity heights, with regard to the selected reference case, is plotted in Fig. 3. The dimensionless time related to the outer shells, which are still expanding together with the universe $(\tau \gtrsim 0)$, exhibits no dependence on overdensity mass. The contrary holds for the inner shells $(\tau \gtrsim 0.5)$ where, for assigned fractional radius, the dimensionless time is an increasing function of the overdensity mass. In other words, with respect to a selected fractional radius, the homogeneous peak collapses "faster" in low-mass overdentity than in large-mass overdensities. The dependence on the mean overdensity height appears to be negligible.

The dimensionless distance, $\alpha=\alpha_{c}\left(r_{i}\right)=r\left(t_{c}\right) / r_{\max }\left(r_{i}\right)$, as a function of the fractional radius, $\xi=r / R$, at the end of central collapse, for different masses and mean overdensity heights, with regard to the selected reference 

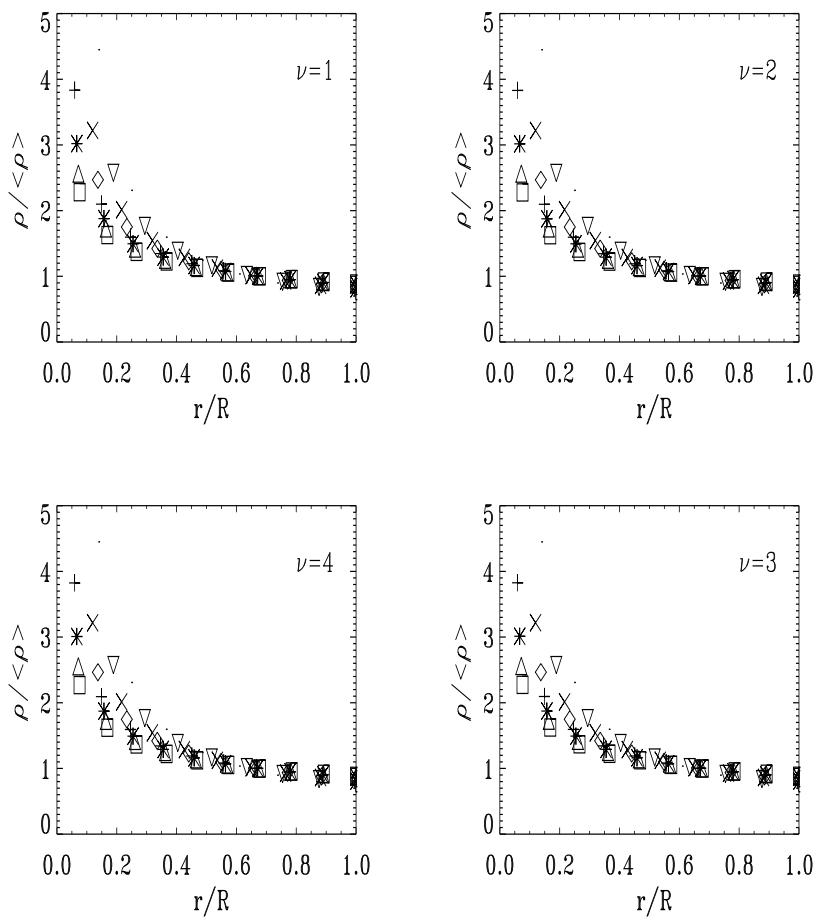

Figure 1: The fractional density, $\rho(r) / \bar{\rho}=\rho(r) /\langle\rho\rangle$, as a function of the fractional radius, $\xi=r / R$, at the end of central collapse, for different masses and mean overdensity heights, $\bar{\nu}_{i}=\nu$, with regard to the selected reference case. Different symbols correspond to the following values of $\log \left(M / \mathrm{M}_{10}\right)$ : -1 (squares); 0 (triangles); 1 (asterisks); 2 (Greek crosses); 3 (diamonds); 4 (St. Andrew's crosses); 5 (reversed triangles); 6 (dots). 

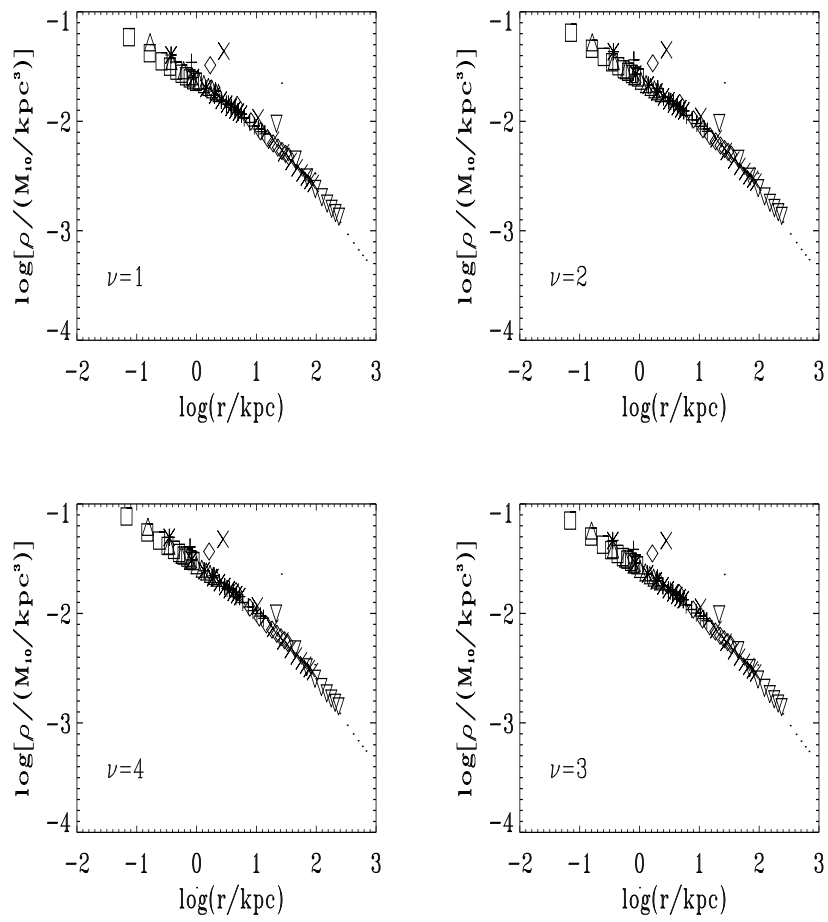

Figure 2: The decimal logarithm of the density, $\log \left[\rho /\left(\mathrm{M}_{10} / \mathrm{kpc}^{3}\right)\right]$, as a function of the decimal logarithm of the radius, $\log (r / \mathrm{kpc})$, at the end of central collapse, for different masses and mean overdensity heights, $\bar{\nu}_{i}=\nu$, with regard to the selected reference case. Caption of symbols as in Fig. 1. 

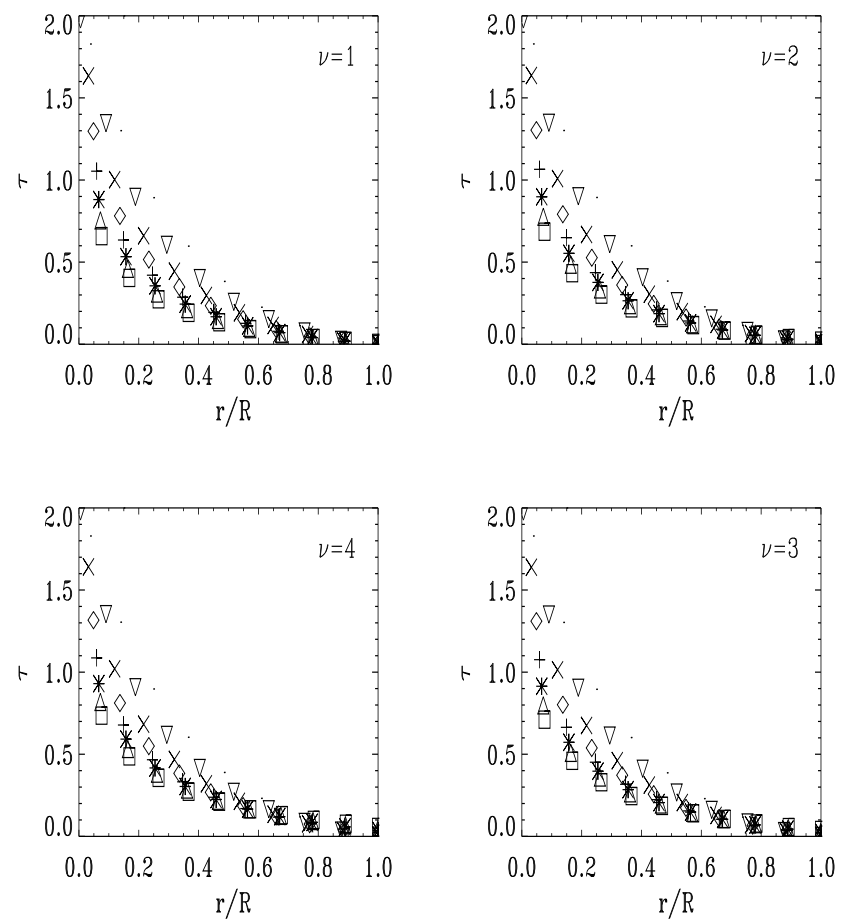

Figure 3: The dimensionless time, $\tau=\tau_{c}\left(r_{i}\right)=t_{c} / t_{\max }\left(r_{i}\right)$, as a function of the fractional radius, $\xi=r / R$, at the end of central collapse, for different masses and mean overdensity heights, with regard to the selected reference case. Caption of symbols as in Fig. 1. 

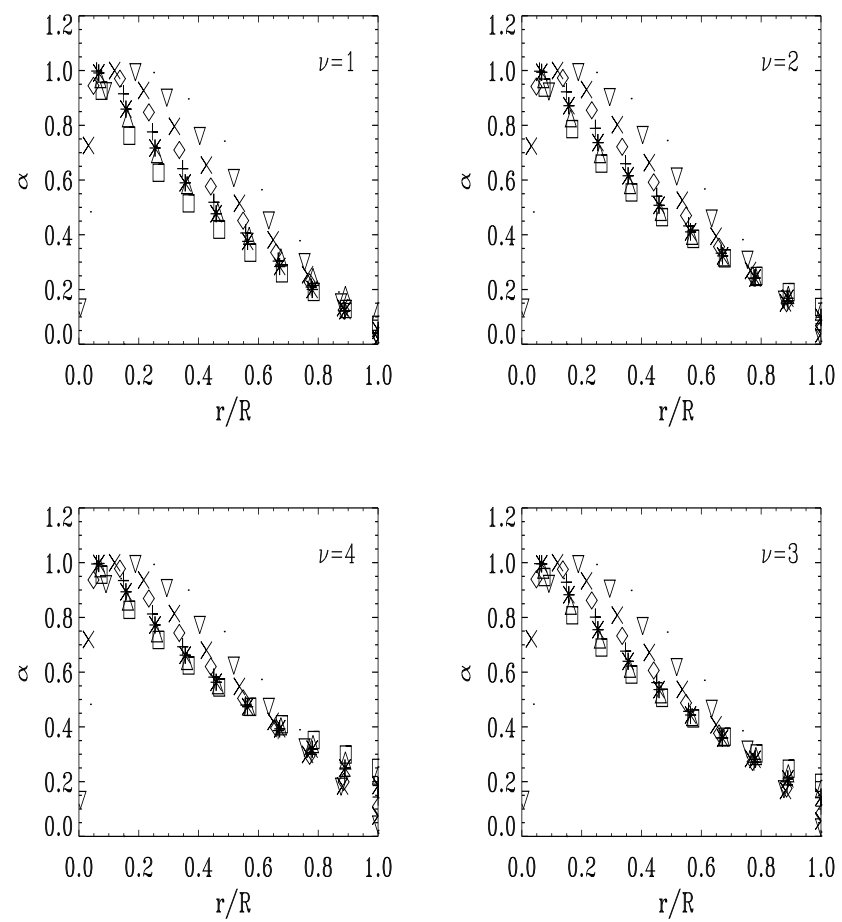

Figure 4: The dimensionless distance, $\alpha=\alpha_{c}\left(r_{i}\right)=r\left(t_{c}\right) / r_{\max }\left(r_{i}\right)$, as a function of the fractional radius, $\xi=r / R$, at the end of central collapse, for different masses and mean overdensity heights, with regard to the selected reference case. Caption of symbols as in Fig. 1.

case, is plotted in Fig. 4. The dimensionless distance related to the outer shells, which are still expanding together with the universe $(\alpha \gtrsim 0)$, is a decreasing function of the overdensity mass, according to the initial conditions via Eq. (86). The above mentioned effect is less evident moving inwards, until no appreciable mass dependence is shown. The trend is reversed for the still expanding inner shells where the dimensionless distance is an increasing function of the overdensity mass. For fixed fractional radius, $r\left(t_{c}\right) / R\left(t_{c}\right)$, the inner shells are closer to maximum expansion, or end of contraction, for large-mass overdensities with respect to low-mass overdensities. In other words, for shells with fixed fractional radius, the homogeneous peak collapses "faster" in low-mass overdensities with respect to large-mass overdensities: 
at the end of central collapse, shells which are turning around correspond to a smaller initial fractional radius for low-mass overdensities, with respect to large-mass overdensities. The fractional radius at the end of central collapse, $\xi=r / R$, where the fractional distance, $\alpha$, shows no appreciable dependence on the overdensity mass, is a decreasing function of the mean overdensity height, passing from $\xi \approx 0.9$ for $\bar{\nu}_{i}=1$ to $\xi \approx 0.7$ for $\bar{\nu}_{i}=4$.

The effect of a change of the model parameters on the results, is shown in Fig. 5 for the dimensionless time, $\tau=\tau_{c}\left(r_{i}\right)=t_{c} / t_{\max }\left(r_{i}\right)$, as a function of the fractional radius, $\xi=r / R$, at the end of central collapse, and in Fig. 6 for the dimensionless distance, $\alpha=\alpha_{c}\left(r_{i}\right)=r\left(t_{c}\right) / r_{\max }\left(r_{i}\right)$, as a function of the fractional radius, $\xi=r / R$, at the end of central collapse, respectively, for different masses. The selected reference case is placed on the middle right, which is the same as in Fig. 3 and Fig. 4 (bottom right), respectively. The changes in parameter values with respect to the selected reference case, are listed on each panel.

The limiting situation of homogeneous initial density profiles, $b=3$, is shown for comparison only, as it would imply a cosmic time at the end of central collapse, longer than the age of the universe for large-mass overdensities $\left(M / \mathrm{M}_{10} \geq 10^{3}\right)$. Slopes within a fiducial range, $2.85 \leq b \leq 2.95$, make little change on both the dimensionless time and the dimensionless distance, as shown in Figs. 5 and 6. A weak trend is exhibited, where the evolution of different shells is closer in shallower density profiles and vice versa, as expected.

According to the assumed hole-hosting dark matter halo mass relation, Eq. (89), the hole mass is larger (for assigned hosting dark matter halo mass) for larger exponent, $\beta$, and/or redshift, $z$, and vice versa, which implies closer evolution of different shells for low-mass holes and vice versa. An inspection of Figs. 5 and 6 shows that it is the case, and the related trend is more visible than in the case of changing slope of the initial density profile, $b$.

In the light of the model presented in the current attempt, holes are the first structures which form in proto-galaxies, when the upper shells are still collapsing or expanding. 

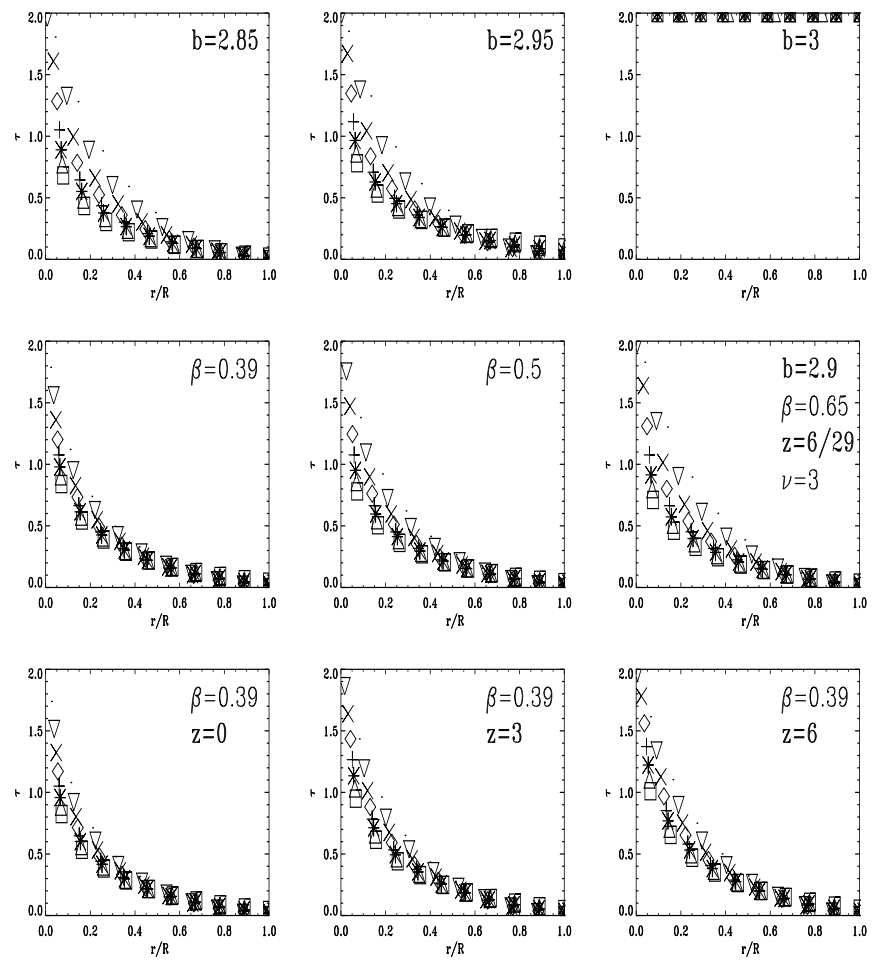

Figure 5: The dimensionless time, $\tau=\tau_{c}\left(r_{i}\right)=t_{c} / t_{\max }\left(r_{i}\right)$, as a function of the fractional radius, $\xi=r / R$, at the end of central collapse, for different masses, mean overdensity height $\nu=\bar{\nu}=3$, and different slopes of the initial density profile, $b$, Eq. (5), exponent, $\beta$, and redshift, $z$, appearing in the assumed hole-hosting dark matter halo mass relation, Eq. (89). The selected reference case is placed on the middle right (same as in Fig. 3, bottom right). The parameter values which have been changed with respect to the reference case, are listed on each panel. The limiting situation of homogeneous initial density profiles, $b=3$, is shown for comparison only. Caption of symbols as in Fig. 1. 

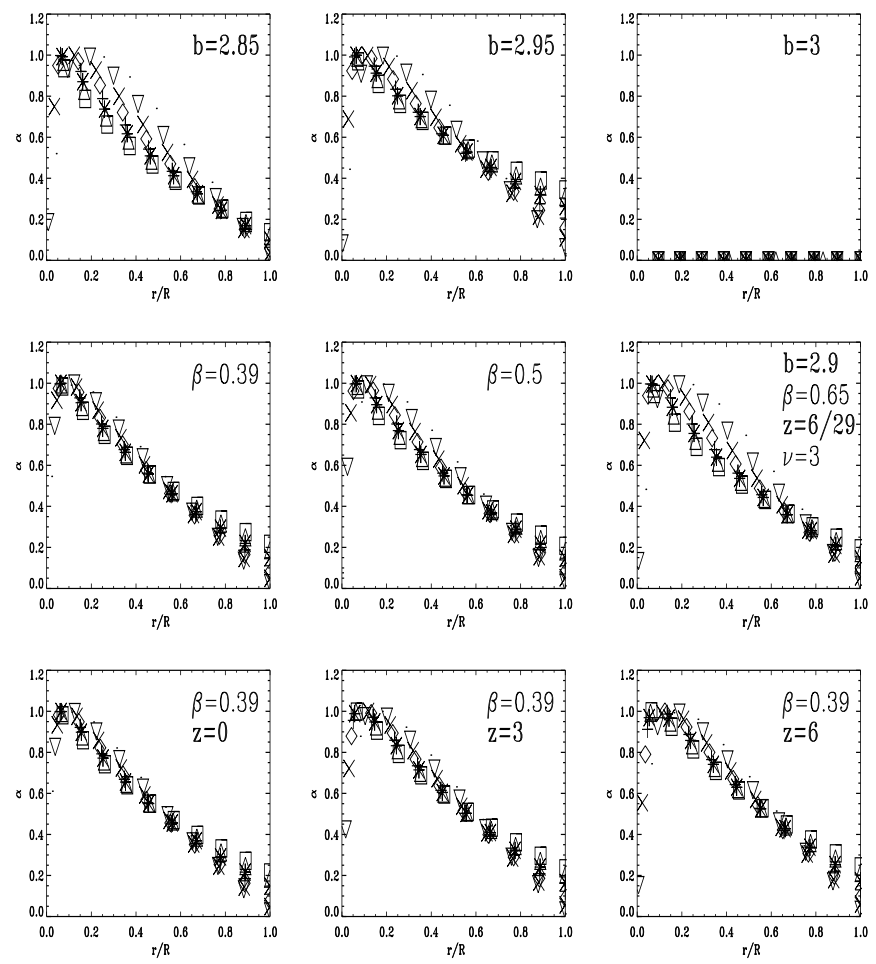

Figure 6: The dimensionless distance, $\alpha=\alpha_{c}\left(r_{i}\right)=r\left(t_{c}\right) / r_{\max }\left(r_{i}\right)$, as a function of the fractional radius, $\xi=r / R$, at the end of central collapse, for different masses, mean overdensity height $\nu=\bar{\nu}=3$, and different slopes of the initial density profile, $b$, Eq. (5), exponent, $\beta$, and redshift, $z$, appearing in the assumed hole-hosting dark matter halo mass relation, Eq. (89). The selected reference case is placed on the middle right (same as in Fig. 4, bottom right). The parameter values which have been changed with respect to the reference case, are listed on each panel. The limiting situation of homogeneous initial density profiles, $b=3$, is shown for comparison only. Caption of symbols as in Fig. 1. 


\section{Discussion}

Gravitational collapse may be a viable mechanism for hole generation, due to two orders of reasons. First, remnants of sufficiently massive Type II supernovae are thought to be black holes (e.g., Nomoto et al. 2005; Ohkube et al. 2006). Second, the mean density of a (spherical-symmetric) black hole is a decreasing function of the gravitational radius. Low-mass $\left(m \lesssim 20 \mathrm{~m}_{\odot}\right.$ ) Type II supernovae leave a neutron (or more exotic) star remnant, which is sustained by the Fermi pressure within a radius about three times larger than the gravitational radius. The Fermi pressure makes the collapsing core bounce at a density about twice the nuclear density, and later attain a virialized configuration. On the other hand, the bounce would occur inside the gravitational radius for more massive $\left(m \approx 20-25 \mathrm{~m}_{\odot}\right)$ Type II supernovae, which implies black hole formation. If (nonbaryonic) dark matter is mainly made of fermions, a similar mechanism could hold. Low-mass $\left(M \lesssim 10 \mathrm{M}_{10}\right)$ dark matter overdensities could leave a fermion ball remnant at the end of central collapse, while larger masses could imply hole generation (e.g., Viollier 1994; Munyaneza and Viollier 2002; Munyaneza and Biermann 2006).

Radial motions are better preserved in collisional fluids where energy dissipation takes place, such as in supernovae, than in collisionless fluids where no energy dissipation occurs, such as dark matter overdensities. Clump formation and tidal interactions between clumps or from neighbourhing overdensities, convert ordered radial motions into random orbital motions. On the other hand, the local maximum at the overdensity deep interior, is a very special place where both tidal interactions and acquisition of angular momentum may safely be neglected. Accordingly, radial motions could be preserved up to hole formation.

The extrapolation of the hole-hosting dark matter halo mass relation, Eq. (89), to early cosmic times, has been considered as the most viable expression of the homogeneous peak to overdensity mass ratio. The related value could be overstimated if only the inner part of the homogeneous peak is the hole progenitor, and understimated if substantial mass accretion onto the hole works. The extrapolation of Eq. (89) in the above mentioned sense, implies the mass of the hole progenitor is proportional to the overdensity mass, at fixed redshift. If the total clump number or the typical clump mass does not appreciably depend on the overdensity mass, clumps within low- 
mass overdensities are expected to be efficient in exerting tidal actions and related departure from radial motions. Accordingly, central collapse and hole formation could be inhibited, in alternative to the formation of a fermion ball.

Though the assumed density profile at the beginning of evolution, Eq. (5), has no physical motivation, still it cannot be considered as completely ad hoc. In fact, it is lying between two limiting situations, related to a homogeneous and a generalized Roche sphere (a homogeneous sphere surrounded by a massless atmosphere), respectively. It is defined by two parameters: a powerlaw exponent, $b$, and a fractional mass, $\kappa=M_{\mathrm{pk}} / M_{\mathrm{tr}}$. The special cases, $b=0$ and $b=3$, are related to generalized Roche and homogeneous spheres, respectively. A fiducial range, $2.85 \leq b \leq 2.95$, has been chosen for the following reasons: low values $(b<2.85)$ would imply exceedingly high peak overdensities, while large values $(b>2.95)$ would imply exceedeingly long cosmic times at the end of central collapse. The main feature is the presence of a homogeneous peak which turns around and collapses into a black hole, and an inhomogeneous envelope which, after expansion, collapses and relaxes.

The fractional mass, $\kappa$, has been selected as a function of both the overdensity mass and the redshift, expressed by Eq. (89), provided overdensity and homogeneous peak mass coincide with dark halo and hole mass, respectively. Recent empirical correlations are related to special choices of the parameters in Eq. (89), namely: $(\beta, z)=(0.65,6 / 29)$ (Ferrarese, 2002); $(\beta, z)=(0.27,19 / 58)$ (Baes et al., 2003); $\beta=0.39$ (Shankar and Mathar, 2007). Also, Eq. (89) is close to its counterpart calibrated locally through statistical arguments (Shankar et al., 2006) and to its counterpart obtained using semianalytical models (Granato et al., 2004; Lapi et al., 2006). In conclusion, the assumed density profile at the beginning of evolution, Eq. (5), may be considered as a phenomenological one.

Strictly speaking, the model discussed in the current attempt holds for isolated overdensities in a secondary infall scenario, where the inner and denser regions first virialize while the outer and less dense regions are still expanding (Gunn 1977). On the other hand, the formation of dark haloes is characterized by accretion of smaller subunits or merger between systems of comparable size, and identification of dark matter haloes in simulations appears problematic (e.g., Bett et al. 2007). Unexpectedly, a recent investigation shows that the secondary infall scenario provides a valid theoretical framework for calculating the structure and evolution of dark matter haloes in an expanding universe, and that its predictions with respect to the density 
profiles are in close agreement with full $\mathrm{N}$-body simulations (Ascasibar et al. 2007). In this view, most of the diversity in density profiles is contributed by the scatter in their primordial counterparts rather the scatter in angular momentum (Ascasibar et al. 2007) i.e. the nature of the initial conditions rather than the details of the evolution.

Though hole gravitational radius cannot be resolved by numerical simulations, the resulting dark halo density profile appears to be cusped at the centre (e.g., Navarro et al. 1995, 1996; Moore et al. 1998, 1999; Fukushige and Makino 2001, 2003; Diemand et al. 2004; Reed et al. 2005), but recent results also allow cored density profiles (e.g., Fukushige and Makino 2004; Navarro et al. 2004; Merritt et al. 2005). If the presence of a central cusp in simulated dark matter haloes is a real effect instead of an artefact due to computer codes, a physical interpretation could be hole formation at the end of central collapse.

Central collapse within local density maxima is one way of avoiding the problem of haloes at high redshifts $(z \approx 6)$ that have had insufficient time to grow at the Salpeter rate from solar or intermediate mass black hole progenitors (e.g., Willott et al., 2003; Miller et al., 2006). In this view, holes are the first structures formed in evolving overdensities, while proto-haloes, proto-bulges, and proto-disks are still relaxing. The active (quasar) phase begins just after hole formation, when the outstanding shells are still collapsing, and may occasionally be enhanced by merger events. The cosmic time at the end of central collapse does not exceed a few hundredths of Gyr, see Tab. 4, in agreement with observations of high-redshift quasars. Virialization takes place later, at a cosmic time equal to about $3 \mathrm{Gyr}$, or $z \approx 2$, provided $M / \mathrm{M}_{10}=500$ [deduced from Cromm et al., 2005, using $h=0.65$ in accordance with Eq. (35)] and $\bar{\nu}=3$ are typical for luminous quasars, see again Tab. 4. The occurrence of virialization, gas exhaustion due to star formation, and absence of merger events in the hosting spheroid component, mark the beginning of the quiescent phase.

The simple model used in the current attempt deals with isolated overdensities where neither accretion nor merging occurs. Hole formation via dark matter collapse implies a limited range of mass, where the lower limit is related to the stability of a fermion ball (provided dark matter is mainly made of fermions) or to the occurrence of tidal effects between clumps, and the upper limit is related to the end of central collapse at a cosmic time equal to the age of the universe. Accordingly, very massive holes are expected 
within clusters of galaxies and superclusters. Though the model is limited to hole formation, still some qualitative considerations on the evolution can be performed.

The picture of holes forming at high redshifts and remaining largely unchanged since then, is not consistent with the observed luminosity density of active galactic nuclei and the inferred local hole density, unless the radiative efficiency of the luminosity of active galactic nuclei is unfeasibly high (Marconi et al., 2004). So it appears that holes have been continuing to accrete mass during the cosmic epochs in which dark matter haloes and their associated galaxies have also continued to be assembled.

In the light of the model used in the current paper, the above scenario still holds with regard to the baryonic precursor of the hole mass budget. On the other hand, little change occurs since hole formation with regard to the (dominant) nonbaryonic precursor. In fact, the basic assumption is that seed holes are created at the end of central collapse $(z>10)$ instead of in major mergers which, in addition, trigger an episode of gas accretion in overdensities with a pre-existing hole. In other words, mass accretion occurs after hole formation, while in earlier attempts mass accretion occurs during hole formation (e.g., Di Matteo et al., 2003; Bromley et al., 2004; Miller et al., 2006; Lapi et al., 2006; Li et al., 2007). Baryon accretion may highly be reduced by central starburst or radiative feedback (e.g., Ciotti and Ostriker, 2007). Nonbaryonic matter accretion may not significantly increase hole mass, due to the lack of a mechanism to dissipate angular momentum, and it may be important only in early times, $z \gtrsim 30$ (Mack et al., 2007).

Several previous models have considered the possibility that holes form from low-mass black holes related to pop. II (e.g., Haiman and Loeb, 2001) or pop. III (e.g., Volonteri et al., 2003) stars. However, there are various problems associated with the above mentioned scenarios (e.g., Haehnelt, 2003; Pelupessy et al., 2007). More specifically, some mechanism is required to facilitate the migration of seed black holes to the centre of their hosting spheroid.

Another class of models focused on the possibility that holes form directly from the collapse of a large gaseous cloud placed in the central overdensity region (e.g., Rees, 1984; Haehnalt and Rees, 1993; Silk and Rees, 1998; Bromley et al., 2004), where the problem is the need to avoid fragmentation and related departure from radial motions during the collapse. In particular, it is assumed (Bromley et al., 2004) that the collapse is also connected to 
overdensity major mergers, in the sense that the same tidally stripped gas which falls into the centre of a post-merger overdensity and can fuel quasar accretion, is also viewed as the source of the initial hole formation.

The model used in the current paper follows the same line of thought but is different to many respects, namely: (i) hole formation is (mainly) due to nonbaryonic matter instead of gas; (ii) the initial driving mechanism is contraction of local density maxima after turnaround instead of major overdensity merger; (iii) quasar accretion is initially fuelled by gas secondary infall instead of tidally stripped gas during overdensity major mergers.

As reported in an earlier attempt (Bromley et al., 2004), any potential model of quasar and active galactic nucleus formation finds itself faced with three major unknowns. First: where, how and with what mass do the initial holes form? Secondly: what events trigger their subsequent fuelling and growth, how much fuel do they supply and how efficiently is it converted into radiative energy? Thirdly: how does feedback (from star formation or the active galactic nucleus activity itself) regulate and possibly even check their growth?

The current paper answers the first point raised above: hole formation is the result of central collapse in local density maxima, mainly due to nonbaryonic dark matter. Subsequent fueling, growth, and feedback, are due to baryonic matter, the sole which can radiate and make quasars and active galactic nuclei shining. On the contrary, the hole mass budget is mainly determined by its nonbaryonic precursor. In this view, current models on active galactic nuclei formation and co-evolution with hosting galaxies, are expected still to hold with regard to the second and the third point raised above.

\section{Conclusion}

Unsustained matter distributions unescapely collapse unless fragmentation and centrifugal or pressure support take place. Starting from the above evidence, supermassive compact objects at the centre of large-mass galaxies (defined as "holes") have been conceived as the end-product of the gravitational collapse of local density maxima (defined as "central collapse") around which positive density perturbations (overdensities) are located. At the beginning of evolution, assumed to occur at recombination epoch, local density 
maxima have been idealized as homogeneous peaks, while the surrounding envelopes have been described by a power-law density profile, $\rho(r) \propto r^{b-3}$, $0 \leq b \leq 3$, where $b=0$ represents a massless atmosphere and $b=3$ a homogeneous layer. The dependence of the density profile on a second parameter, chosen to be the ratio between peak and total (truncated) mass, $\kappa=M_{\mathrm{pk}} / M_{\mathrm{tr}}$, has been analysed.

Overdensity evolution has been discussed in the context of quintessence cosmological models, which should be useful in dealing with the virialized phase. Aiming to describe the central collapse, further investigation has been devoted to a special case where the quintessence effect is equivalent to additional curvature $(w=-1 / 3)$, and overdensities exhibit the selected density profile at recombination epoch. A redshift-dependent, power-law relation between hole and (nonbaryonic) dark halo mass has been used to express the dependence of the fractional mass, $\kappa=M_{\mathrm{pk}} / M_{\mathrm{tr}}$, on the overdensity mass, $M=M_{\mathrm{tr}}$, where the homogeneous peak and overdensity mass are related to the hole and dark halo mass, respectively.

Computations have been performed for a wide range of masses, $-1 \leq$ $\log \left(M / \mathrm{M}_{10}\right) \leq 6$, and mean overdensity heights, $1 \leq \bar{\nu}_{i} \leq 4$, up to the end of central collapse, for the following quantities: homogeneous peak overdensity, $\delta_{\mathrm{pk}}$; turnaround radius, $r_{\max }$; cosmic time at the end of central collapse, $t_{c}$; density profile, $\rho\left[r\left(t_{c}\right)\right]$; logarithmic density profile, $\log \left[\rho /\left(\mathrm{M}_{10} / \mathrm{kpc}^{3}\right)\right]$; ratio of cosmic time at the end of central collapse to turnaround time of the related shell, $\tau_{c}=t_{c} / t\left[r_{\max }\left(r_{i}\right)\right]$; ratio of shell radius at the end of central collapse to shell radius at turnaround, $\alpha_{c}=r\left(t_{c}\right) / r_{\max }\left(r_{i}\right)$. With regard to the last two above mentioned quantities, additional computations have been performed for different slopes of the initial density profile, $b$, and different parameters appearing in the hole-hosting dark matter halo mass relation, $\beta$ and $z$, Eq. (89).

The central collapse has been found to end in early times, no longer than a few hundredths of Gyr, which implies hole formation when proto-haloes, proto-bulges, and proto-disks are still relaxing. No appreciable change has been found in the evolution (up to the end of central collapse) of different mean overdensity heights related to equal masses. On the other hand, it has been recognized that homogeneous peaks collapse (in dimensionless coordinates) "faster" with respect to surroundings envelopes, in low-mass overdensities than in large-mass overdensities. In conclusion, it has been inferred that gravitational collapse of homogeneous peaks within overdensities 
may be a viable mechanism for hole generation.

\section{Acknowledgements}

Thanks are due to S. Masiero for fruitful discussions. The author is indebted with M. Yu. Khlopov for informations on his and coworkers' quoted papers about black hole generation via topological defects during the inflation epoch. The author is deeply grateful to an anonymous referee for critical comments which made substantial improvement to an earlier version of the paper.

\section{References}

[1] Adams, F.C., Bloch, A.M., 2006. ApJ 653, 905.

[2] Ambartsumian, V., 1958. In La Structure et l'Evolution de l'Universe, ed. Institut International de Physique Solvay, p. 267.

[3] Ambartsumian, V., 1965. In The Structure and Evolution of Galaxies, ed. Interscience Publishers, p. 1.

[4] Amendola, L., 2000. PhRvD 62, 043511.

[5] Andriani, E., Caimmi, R., 1991. ApSS 182, 261.

[6] Andriani, E., Caimmi, R., 1994. A\&A 289, 1.

[7] Ascasibar, Y., Hoffman, Y., Gottlöber, S., 2007. MNRAS 376, 393.

[8] Babelberg, S., Shapiro, S.L., 2002. PhRvL 88, 101301.

[9] Baes, M., Buyle, P., Hau, G.K.T., Dejonghe, H., 2003. MNRAS 341, L44.

[10] Basilakos, S., 2003. ApJ 590, 636.

[11] Basilakos, S., Voglis, N., 2007. MNRAS 374, 269.

[12] Battye, R.A., Weller, J., 2003. PhRvD 68, 083506.

[13] Begelman, M.C., Rees, M.J., 1978. MNRAS 185, 847. 
[14] Bett, P., et al., 2007. MNRAS 376, 215.

[15] Bromley, J.M., Somerville, R.S., Fabian, A.C., 2004. MNRAS 350, 456.

[16] Brosche, P., Caimmi, R., Secco, L., 1983. A\&A 125, 338.

[17] Caimmi, R., 1989. A\&A 223, 29.

[18] Caimmi, R., 1990. A\&A 239, 7.

[19] Caimmi, R., 2006. Appl. Math. Comput. 174, 447.

[20] Caimmi, R., 2007. NewA 12, 327.

[21] Caimmi, R., Secco, L., Brosche, P., 1984. A\&A 139, 411.

[22] Caimmi, R., Secco, L., Andriani, E., 1990. ApSS 168, 131.

[23] Caimmi, R., Secco, L., 1992. ApJ 395, 119.

[24] Caimmi, R., Marmo, C., 2003. NewA 8, 119.

[25] Caimmi, R., Marmo, C., 2004. SerAJ 169, 11.

[26] Caimmi, R., Marmo, C., Valentinuzzi, T., 2005. SerAJ 170, 13.

[27] Caldwell, R.R., Dave, R., Steinhardt, P.J., 1998a. PhRvL 80, 1582.

[28] Caldwell, R.R., Dave, R., Steinhardt, P.J., 1998b. ApSS 261, 303.

[29] Carroll, S.M., Press, W.H.., Turner, E.L., 1992. ARA\&A 30, 499.

[30] Cavaliere, A., Morrison, P., Pacini, F., 1970. ApJ 162, L133.

[31] Cavaliere, A., Pacini, F., Setti, G., 1969. ApL 4, 103.

[32] Ciotti, L., Ostriker, J.P., 2007. ApJ 665, 1038.

[33] Colgate, S.A., 1967. ApJ 150, 163.

[34] David, L.P., Durisen, R.H., Cohn, H.N., 1987a. ApJ 313, 556.

[35] David, L.P., Durisen, R.H., Cohn, H.N., 1987b. ApJ 316, 505. 
[36] Diemand, J., Moore, B., Stadel, J., 2004. MNRAS 353, 624.

[37] Di Matteo, T., Croft, R.A.C., Springel, V., Hernquist, L., 2003. ApJ $593,56$.

[38] Doran, M., Schmidt, J.-M., Wetterich, C., 2001. PhRvD 64, 123520.

[39] Duncan, M.J., Shapiro, S.L., 1983. ApJ 268, 565.

[40] Ferrarese, L., 2002. ApJ 578, 90.

[41] Ferrarese, L., Ford, H., 2005. Space Sci. Rev. 116, 523.

[42] Fukushige, T., Makino, J., 2001. ApJ 557, 533.

[43] Fukushige, T., Makino, J., 2003. ApJ 588, 674.

[44] Fukushige, T., Kawai, A., Makino, J., 2004. ApJ 606, 625.

[45] Granato, G.L., De Zotti, G., Silva, L., et al., 2004. ApJ 650, 42.

[46] Gunn, J.E., 1977. ApJ 218, 592.

[47] Gunn, J.E., 1987. The Galaxy, NATO ASI Ser. C207,

[48] Haehnelt, M.G., 2003. In Ho, L.C., ed, Carnegie Observatories Astrophys. Ser. Vol. I, Coevolution of Black Holes and Galaxies, Cambridge Univ. Press, Cambridge (astro-ph/0307378).

[49] Haehnelt, M.G., Rees, M.J., 1993. MNRAS 263, 168.

[50] Haiman, Z., Loeb, A., 2001. ApJ 552, 459.

[51] Heavens, A.F., Peacock, J.A., 1988. MNRAS 232, 339.

[52] Hernquist, L., 1990. ApJ 356, 359.

[53] Hiotelis, N., 2003. MNRAS 344, 149.

[54] Horellou, C., Berge, J., 2005. MNRAS 360, 1393.

[55] Iliev, I.T., Shapiro, P.R., 2001. MNRAS 325, 468. 
[56] Johnson, J.L., Bromm, V., 2007. MNRAS 374, 1557.

[57] Khlopov, M.Yu., Rubin, S.G., Sakharov, A.S., 2002. Grav. \& Cosmol. 8, Suppl., 57.

[58] Khlopov, M.Yu., Rubin, S.G., 2004. Cosmological Pattern of Microphysics in the Inflationary Universe, Kluver Academic Publishers, Dordrecht.

[59] Khlopov, M.Yu., Rubin, S.G., Sakharov, A.S., 2005. Astropart. Phys. $23,265$.

[60] Koushiappas, S.M., Bullock, J., Dekel, A., 2004. MNRAS 354, 292.

[61] Landau, L., Lifchitz, E., 1966. Theorie du champs, Mir, Moscow.

[62] Lapi, A., Shankar, F., Mao, J., et al., 2006. ApJ 650, 42.

[63] Li, Y., Hernquist, L., Robertson, B., et al., 2007. ApJ 665, 187.

[64] Limber, D.N., 1959. ApJ 130, 414.

[65] Maccarone, T.J., Kundu, A., Zepf, S.E., et al., 2007. Nature 445, 183.

[66] Mack, K.J., Ostriker, J.P., Ricotti, M., 2007. ApJ 665, 1277.

[67] Madau, P., Rees, M.J., 2001. ApJ 551, L27.

[68] Maio, U., Dolag, K., Meneghetti, M., et al., 2006. MNRAS 373, 869.

[69] Manera, M., Mota, D.F., 2006. MNRAS 371, 1373.

[70] Maor, E., 1998. ApJ 494, L181.

[71] Maor, I., Lahav, O., 2005. JCAP 7, 3.

[72] Merloni, A., Nayakshin, S., Sunyaev, R.A., eds 2005. Growing Black Holes: Accretion in a Cosmological Context, Springer, Germany.

[73] Merritt, D., Navarro, F.J., Ludlow, A., et al., 2005. ApJ 624, L85.

[74] Merritt, D., 2006. Rep. Prog. Phys. 69, 2513. 
[75] Miller, M.C., 2006. MNRAS 367, L32.

[76] Moore, B., Governato, F., Quinn, T., et al., 1998. ApJ 499, L5.

[77] Moore, B., Quinn, T., Governato, F., et al., 1999. MNRAS 310, 1147.

[78] Morrison, P., 1969. ApJ 157, L73.

[79] Mota, D.F., van de Bruck, C., 2004. A\&A 421, 71.

[80] Munyaneza, F., Viollier, R.D., 2002. ApJ 564, 274.

[81] Munyaneza, F., Biermann, P.L., 2006. A\&A 458, L9.

[82] Navarro, J.F., Frenk, C.S., White, S.D.M., 1995. MNRAS 275, 720.

[83] Navarro, J.F., Frenk, C.S., White, S.D.M., 1996. ApJ 462, 563.

[84] Navarro, J.F., Hayashi, E., Power, C., et al., 2004. MNRAS 349, 1039.

[85] Nomoto, K., Tominaga, N., Umeda, H., et al., 2005. Nucl. Phys. 32, 51.

[86] Nunes, N.J., Mota, D.F., 2006. MNRAS 368, 751.

[87] Ohkubo, T., Umeda, H., Maeda, K., et al., 2006. ApJ 645, 1352.

[88] Peebles, P.J.E., 1980. The Large-Scale Structure of the Universe, Princeton Univ. Press, New Jersey.

[89] Pelupessy, F.I., Di Matteo, T., Ciardi, B., 2007. ApJ 665, 107.

[90] Percival, W.J., 2005. A\&A 443, 819.

[91] Portegies Zwart, S.F., Baumgardt, H., Hut, P., et al., 2004. Nature 428, 724.

[92] Quinlan, G.D., Shapiro, S.L., 1987. ApJ 321, 199.

[93] Quinlan, G.D., Shapiro, S.L., 1989. ApJ 343, 725.

[94] Reed, D., Governato, F., Verde, L., et al., 2005. MNRAS 357, 82.

[95] Rees, M.J., 1966. Nature 211, 468. 
[96] Rees, M.J., 1984. ARA\&A 22, 471.

[97] Rubin, S.G., Sakharov, A.S., Khlopov, M. Yu., 2001. J. Exp. Theor. Phys. 92, 921.

[98] Ryden, B.S., Gunn, J.E., 1987. ApJ 318, 15.

[99] Sanders, R.H., 1970. ApJ 162, 791.

[100] Sersic, J.L., 1968. Atlas de Galaxies Australes, Cordoba: Obs. Astron., Univ. Nac. Cordoba.

[101] Shankar, F., Mathur, S., 2007. ApJ 660, 1051.

[102] Shankar, F., Lapi, A., Salucci, P., et al., 2006. ApJ 643, 14.

[103] Silk, J., 2005. MNRAS 364, 1337.

[104] Silk, J., Rees, M.J., 1998. A\&A 331, L1.

[105] Silveira, V., Wega, I., 1994. PhRvD 64, 4890.

[106] Spaans, M., Silk, J., 2006. ApJ 652, 902.

[107] Spitzer, L.J., Saslaw, W.C., 1966. ApJ 143, 400.

[108] Spitzer, L.J., Stone, M.E., 1967. ApJ 147, 519.

[109] Terrel, J., 1964. Science 145, 918.

[110] Viollier, R.D., 1994. Prog. Part. Nucl. Phys. 32, 51.

[111] Volonteri, M., Haardt, F., Madau, P., 2003. ApJ 582, 559.

[112] Wang, L., Steinhardt, P.J., 1998. ApJ 508, 483.

[113] Wang, L., Caldwell, R.R., Ostriker, J.P., et al., 2000. ApJ 530, 17.

[114] Weinberg, N.N., Kamionkowski M., 2003. MNRAS 341, 251.

[115] Willott, C.J., Mclure, R.J., Jarvis, M.J., 2003. ApJ 587, L15.

[116] Zeldovich, J.B., Novikov, I.D., 1982. Struttura ed Evoluzione dell'Universo, Mir, Moscow.

[117] Zhao, H.S., 1996. MNRAS 278, 488. 


\section{Appendix}

\section{A On the light velocity in vacuum}

Strictly speaking, light travels across the vacuum (intended in philosophical sense, as absence of everything) only in classical (Newton) and specialrelativistic (Einstein) mechanics, where it is conceived as made of corpuscles. If, on the other hand, light is conceived as waves (Huygens), then it propagates across a homogeneous medium called ether. In general-relativistic mechanics (Einstein) ether is replaced by gravitational field of the universe as a whole. In quantum mechanichs (Bohr, de Broglie, Heisemberg, Schrödinger) light can be considered as made of either corpuscles or waves, and the propagation takes place across the quantum void, which hosts some form of energy, and then has not to be intended in the above specified philosophical sense. Light velocity is maximum in vacuum (or ether, or quantum void), while it decreases for increasing refraction index when propagation occurs within a (homogeneous) medium, due to photon interaction with medium constituents.

Light velocity has been deduced from experiments on the Earth or within the solar system at most. According to current QCDM cosmologies, the solar system is embedded within (nonbaryonic) dark matter and dark energy. The current value of the light velocity coincides with the maximum attainable value only if no interaction is assumed to occur between (i) photons and dark matter, and (ii) photons and dark energy. If otherwise, light velocity could be higher in intergalactic voids and/or in early (cosmic) times, where the effect of the dark energy was negligible. Even in pure matter universes, light propagation takes place across the quantum void, where some form of energy is present.

In conclusion, the term "light velocity in vacuum" has to be intended as "light velocity in absence of baryonic matter" under the assumption that photons interact with neither (nonbaryonic) dark matter, nor dark energy.

\section{B The $\left[\mathrm{kpc} \mathrm{M}_{10} \mathrm{Gyr}\right]$ system of measure}

In dealing with large-scale celestial objects, such as galaxies or cluster of galaxies, it may be convenient to use an appropriate system of measure in- 
stead of the standard astrophysical one, [cm g s]. To this aim, unit length, unit mass, and unit time, shall be taken as:

$$
\begin{aligned}
& 1 \mathrm{kpc}=3.0856775806663110^{21} \mathrm{~cm} ; \\
& \mathrm{M}_{10}=10^{10} \mathrm{~m}_{\odot}=1.989110^{43} \mathrm{~g} ; \\
& 1 \mathrm{Gyr}=10^{9} \mathrm{y}=3.153610^{16} \mathrm{~s} ;
\end{aligned}
$$

and the related "mathematical" year is defined as $1 \mathrm{y}=(365 \times 24 \times 60 \times 60)$ s, independent of the Earth revolution around the Sun.

According to Eq. (90), the astronomical unit is:

$$
1 \mathrm{AU}=1.4959787069110^{13} \mathrm{~cm}=4.8481368121010^{-9} \mathrm{kpc} ;
$$

using Eqs. (90) and (92), the velocity unit reads:

$$
\begin{aligned}
& 1 \mathrm{kpc} \mathrm{Gyr}^{-1}=0.9784619421189466 \mathrm{~km} \mathrm{~s}^{-1} ; \\
& 1 \mathrm{~km} \mathrm{~s}^{-1}=1.022012156992443 \mathrm{kpc} \mathrm{Gyr}^{-1} ;
\end{aligned}
$$

and the light velocity in vacuum is:

$$
c=2.9979245810^{5} \mathrm{~km} \mathrm{~s}^{-1}=3.0639153710^{5} \mathrm{kpc} \mathrm{Gyr}^{-1} ;
$$

on the other hand, the value of the gravitation constant is deduced from Eqs. (90)-(92), as:

$$
G=(6.67259 \mp 0.00085) \mathrm{g}^{-1} \mathrm{~cm}^{3} \mathrm{~s}^{-2}=(44927.5 \mp 5.7) \mathrm{M}_{10}^{-1} \mathrm{kpc}^{3} \mathrm{Gyr}^{-2} \text {. }
$$

Using Eqs. (90) and (91), the unit density reads:

$$
\begin{aligned}
& 1 \mathrm{M}_{10} \mathrm{kpc}^{-3}=6.770310^{-22} \mathrm{~g} \mathrm{~cm}^{-3} \\
& 1 \mathrm{~g} \mathrm{~cm}^{-3}=1.477010^{21} \mathrm{M}_{10} \mathrm{kpc}^{-3}
\end{aligned}
$$

and the value of the mean solar density is:

$$
\bar{\rho}_{\odot}=1.411 \mathrm{~g} \mathrm{~cm}^{-3}=2.08310^{21} \mathrm{M}_{10} \mathrm{kpc}^{-3} ;
$$

where $\bar{R}_{\odot}=6.9610^{10} \mathrm{~cm}$ has been assumed.

Using Eqs. (90)-(94), the unit energy and the unit specific energy read:

$$
\begin{aligned}
& 1 \mathrm{M}_{10} \mathrm{kpc}^{2} \mathrm{Gyr}^{-2}=1.904310^{10} \mathrm{~g} \mathrm{~cm}^{2} \mathrm{~s}^{-2} ; \\
& 1 \mathrm{~g} \mathrm{~cm}^{2} \mathrm{~s}^{-2}=5.251210^{-11} \mathrm{M}_{10} \mathrm{kpc}^{2} \mathrm{Gyr}^{-2} ;
\end{aligned} ;
$$




$$
\begin{aligned}
& 1 \mathrm{kpc}^{2} \mathrm{Gyr}^{-2}=9.57387771942411410^{9} \mathrm{~cm}^{2} \mathrm{~s}^{-2} ; \\
& 1 \mathrm{~cm}^{2} \mathrm{~s}^{-2}=1.04450884929429810^{-10} \mathrm{kpc}^{2} \mathrm{Gyr}^{-2} ;
\end{aligned}
$$

similarly, the unit angular momentum and specific angular momentum read:

$$
\begin{gathered}
1 \mathrm{M}_{10} \mathrm{kpc}^{2} \mathrm{Gyr}^{-1}=6.005510^{69} \mathrm{~g} \mathrm{~cm}^{2} \mathrm{~s}^{-1} ; \\
1 \mathrm{~g} \mathrm{~cm}^{2} \mathrm{~s}^{-1}=1.665110^{-70} \mathrm{M}_{10} \mathrm{kpc}^{2} \mathrm{Gyr}^{-1} ; \\
1 \mathrm{kpc}^{2} \mathrm{Gyr}^{-1}=3.01921807702773210^{26} \mathrm{~cm}^{2} \mathrm{~s}^{-1} ; \\
1 \mathrm{~cm}^{2} \mathrm{~s}^{-1}=3.31211583425749010^{-27} \mathrm{kpc}^{2} \mathrm{Gyr}^{-1} ;
\end{gathered}
$$

accordingly, velocities, energies, and angular momenta, may be translated from $[\mathrm{cm} \mathrm{gs}]$ to $\left[\mathrm{kpc} \mathrm{M}_{10} \mathrm{Gyr}\right]$ system of measure and vice versa.

\section{Evolution of bound overdensities in CDM universes}

Overdensity evolution in CDM universes is determined by Eqs. (17), (61), (65), (70), and (77)-(82). To perform calculations, an explicit expression of the function, $g(r)$, defined by Eq. (82b), is needed. To help the reader, the procedure used in earlier attempts (Andriani and Caimmi, 1991, 1994) shall be repeated here for the case of interest i.e. bound perturbations in an open universe.

To this aim, it is useful to define the dimensionless variables (Andriani and Caimmi, 1991):

$$
\begin{aligned}
& \alpha^{\prime}=\frac{r}{r_{i}} ; \quad \tau^{\prime}=\frac{t}{\left(t_{f f}\right)_{i}} ; \\
& \left(t_{f f}\right)_{i}=\left(\frac{\pi^{2}}{8} \frac{r_{i}^{3}}{G\left(M_{m}\right)_{h}}\right)^{1 / 2}=\left(\frac{3 \pi}{32} \frac{1}{G \rho_{h i}}\right)^{1 / 2} ; \quad \alpha_{i}^{\prime}=1 ;
\end{aligned}
$$

(the index, $h$, denotes zero density excess) which are related to their counterparts, expressed by Eqs. (65), via Eq. (74) as:

$$
\alpha=\Delta_{i} \alpha^{\prime} ; \quad \tau=\left(1+\bar{\delta}_{i}\right)^{1 / 2} \Delta_{i}^{3 / 2} \tau^{\prime} ;
$$


with regard to an infinitely thin spherical shell of initial radius, $r_{i}$.

In addition, both the cosmic time, $t$, and the free-fall time at the beginning of evolution, $\left(t_{f f}\right)_{i}$, via Eqs. (74) are independent of $r_{i}$, which implies null first derivatives:

$$
\frac{\partial \tau^{\prime}}{\partial r_{i}}=\frac{\partial \tau_{i}^{\prime}}{\partial r_{i}}=0
$$

Using Eqs. (103), (104), and the trigonometric identity:

$$
\frac{1}{2} \arcsin (1-2 x)+\frac{\pi}{4}=\arcsin \sqrt{1-x} ; \quad 0 \leq x \leq 1 ;
$$

the following expression of Eq. (69) holds:

$$
\begin{aligned}
\tau^{\prime}-\tau_{i}^{\prime}= & \mp \frac{2}{\pi}\left(1+\bar{\delta}_{i}\right)^{-1 / 2} \Delta_{i}^{-3 / 2}\left[-\sqrt{\Delta_{i} \alpha^{\prime}-\left(\Delta_{i} \alpha^{\prime}\right)^{2}}+\sqrt{\Delta_{i}-\Delta_{i}^{2}}\right. \\
& \left.+\frac{1}{2} \arccos \left(1-2 \Delta_{i} \alpha^{\prime}\right)-\frac{1}{2} \arccos \left(1-2 \Delta_{i}\right)\right]
\end{aligned}
$$

where the plus is related to expansion, and the minus to contraction.

The combination of Eqs. (82b) and (103a) yields:

$$
g(r)=\alpha^{\prime}\left[\frac{\partial\left(\alpha^{\prime} r_{i}\right)}{\partial r_{i}}\right]^{-1}=\left(1+\frac{r_{i}}{\alpha^{\prime}} \frac{\partial \alpha^{\prime}}{\partial r_{i}}\right)^{-1}
$$

in terms of the dimensionless radius, $\alpha^{\prime}$.

For spherical-symmetric overdensities, Eq. (20) reads:

$$
\bar{\delta}=\frac{3}{r^{3}} \int_{0}^{r} \delta(r) r^{2} \mathrm{~d} r
$$

and a derivation with respect to $r$ produces:

$$
\frac{\partial \bar{\delta}}{\partial r}=-\frac{3}{r} \bar{\delta}\left(1-\frac{\delta}{\bar{\delta}}\right)
$$

Keeping in mind that the density parameter, $\left(\Omega_{m}\right)_{i}$, is independent of $r_{i}$, a derivation with respect to $r_{i}$ on both sides of Eq. (42b) yields:

$$
\frac{\partial \Delta_{i}}{\partial r_{i}}=\frac{\partial \alpha_{i}}{\partial r_{i}}=-\frac{-1+\Delta_{i}}{1+\bar{\delta}_{i}} \frac{\partial \bar{\delta}_{i}}{\partial r_{i}}
$$


owing to Eqs. (42) and (65a), where the former has been generalized to the sphere bounded by a generic isopycnic surface of initial radius, $r_{i}$.

Using Eqs. (103)-(105) and (107)-(110), a derivation with respect to $r_{i}$ on both sides of Eq. (106), after a lot of algebra, produces the following expression of $g(r)$ :

$$
\begin{aligned}
& {[g(r)]^{-1}=1+3 \frac{1-\Delta_{i}}{\Delta_{i}} \frac{\bar{\delta}_{i}}{1+\bar{\delta}_{i}}\left(1-\frac{\delta_{i}}{\bar{\delta}_{i}}\right)\left\{1-\frac{\Delta_{i}^{2}}{\sqrt{\Delta_{i}-\Delta_{i}^{2}}} \frac{\mp \sqrt{\Delta_{i} \alpha^{\prime}-\left(\Delta_{i} \alpha^{\prime}\right)^{2}}}{\left(\Delta_{i} \alpha^{\prime}\right)^{2}} \times\right.} \\
& \left.\times\left[1+\frac{3 \pi}{4}\left(1+\bar{\delta}_{i}\right)^{1 / 2}\left(\frac{1-\Delta_{i}}{\Delta_{i}^{3}}\right)^{1 / 2}\left(1+\frac{1}{3} \frac{\Delta_{i}}{1-\Delta_{i}}\right)\left(\tau^{\prime}-\tau_{i}^{\prime}\right)\right]\right\} ; \\
& {\left[g\left(r_{i}\right)\right]^{-1}=1}
\end{aligned}
$$

for further details and exhaustive investigation on open, flat, and closed CDM universes hosting bound, zero-energy, and unbound overdensities, refer to Andriani and Caimmi (1991).

Turning again to the earlier dimensionless coordinates, $\alpha$ and $\tau$, the combination of Eqs. (65), (104), and (111) yields Eq. (83). 